\title{
Comparative transcriptome analysis reveals ectopic delta- 5 and delta- 6 desaturases enhance protective gene expression upon Vibrio vulnificus challenge in Tilapia (Oreochromis niloticus)
}

\author{
Pin-Yang Tu', Shin-Jie Huang ${ }^{2}$, Venugopal Rajanbabu ${ }^{3}$, Jen-Leih Wu ${ }^{2}$ and Jyh-Yih Chen ${ }^{1,4^{*}}$
}

\begin{abstract}
Background: Tilapia (Oreochromis niloticus) cultures are frequently infected by Vibrio vulnificus, causing major economic losses to production units. Previously, tilapia expressing recombinant delta-5 desaturase and delta-6 desaturase (D56) were found to be resistant to $V$. vulnificus infection. In this report, we profile the D56-mediated molecular changes underlying this resistance in tilapia. A comparative transcriptome analysis was performed on $V$. vulnificus-infected wildtype and D56-transgenic tilapia using Illumina's sequencing-by-synthesis approach. Gene enrichment analysis on differentially expressed unigenes was performed, and the expression patterns were validated by real-time PCR.

Results: Comparative transcriptome analysis was performed on RNA-sequence profiles obtained from wild-type and D56transgenic tilapia at 0, 6 and $24 \mathrm{~h}$ post-infection with V. vulnificaus. GO and KEGG gene enrichment analyses showed that D56 regulates several pathways and genes, including fatty acid (FA) metabolism associated, and inflammatory and immune response. Expression of selected FA metabolism-associated, inflammatory and immune responsive genes was validated by qPCR. The inflammatory and immune responsive genes that are modulated by FA-associated D56 likely contribute to the enhanced resistance against $V$. vulnificus infection in Tilapia.

Conclusions: Transcriptome profiling and filtering for two-fold change variation showed that 3795 genes were upregulated and 1839 genes were downregulated in D56-transgenic tilapia. These genes were grouped into pathways, such as FA metabolism, FA elongation, FA biosynthesis, biosynthesis of unsaturated FA, FA degradation, inflammation, immune response, and chemokines. FA-associated genes and immune-related genes were modulated by D56 at $6 \mathrm{~h}$ and $24 \mathrm{~h}$ post infection with $\mathrm{V}$. vulnificus. The expression patterns of FA-related genes, inflammatory genes, antimicrobial peptide genes and immune responsive genes at $0,3,6,12,24$ and $48 \mathrm{~h}$ post-infection suggests these genes are involved in the enhanced resistance of D56 transgenic tilapia to $V$. vulnificus.
\end{abstract}

Keywords: Delta-6 desaturase and delta-5 desaturase (D56), D56 transgenic tilapia fish, RNA-seq, Fatty acid-associated genes, Immune responsive genes, Inflammatory genes

\footnotetext{
* Correspondence: zoocjy@gate.sinica.edu.tw

'Marine Research Station, Institute of Cellular and Organismic Biology,

Academia Sinica, 23-10 Dahuen Rd., Jiaushi, Ilan 262, Taiwan

${ }^{4}$ The iEGG and Animal Biotechnology Center, National Chung Hsing University, Taichung 402, Taiwan

Full list of author information is available at the end of the article
}

(C) The Author(s). 2021 Open Access This article is licensed under a Creative Commons Attribution 4.0 International License, which permits use, sharing, adaptation, distribution and reproduction in any medium or format, as long as you give appropriate credit to the original author(s) and the source, provide a link to the Creative Commons licence, and indicate if changes were made. The images or other third party material in this article are included in the article's Creative Commons licence, unless indicated otherwise in a credit line to the material. If material is not included in the article's Creative Commons licence and your intended use is not permitted by statutory regulation or exceeds the permitted use, you will need to obtain permission directly from the copyright holder. To view a copy of this licence, visit http://creativecommons.org/licenses/by/4.0/ The Creative Commons Public Domain Dedication waiver (http://creativecommons.org/publicdomain/zero/1.0/) applies to the data made available in this article, unless otherwise stated in a credit line to the data. 


\section{Background}

Tilapia (Oreochrombis niloticus) is an important commercial aquaculture species throughout the world, and its production is severely affected by the pathogenic bacteria Vibrio vulnificus, which causes septicemia in fish and humans [1-4]. Omega-3 polyunsaturated fatty acids (n-3 PUFAs) are known to exert beneficial effects, such as protection of liver, reduction of cholesterol, lower blood pressure and protect from cardiovascular diseases $[5,6]$. Furthermore, n-3 PUFAs show positive ionotropic effects and minimize tachyarrhythmia in animal models [7]. Many of these effects may be mediated by alterations in the proinflammatory cytokines, TNF- $\alpha$, IL-1 $\beta$, IL-6, prostaglandin (PG) E2, and PGF1 $\alpha$, which modulate the immune response in model organisms [8-10]. Dietary supplementation with eicosanoids and n-3 PUFAs is well documented to affect immune cell function and B-cell activity [11, 12], and a recent report showed that PUFA-rich food limit pathogen infection in the aquatic organisms [13]. Similarly, transgenic expression of n-3 PUFA biosynthesis genes from Atlantic salmon, i.e., Fatty acyl desaturase synthase delta (Fadsd) 5 and Fadsd6, in zebrafish limits infection with Vibrio alginolyticus and $V$. vulnificus $[5,14]$.

Previously we reported the dual expression of SsFadsd5 and SsFadsd6 (D56) in tilapia [15]. The dual expression of these genes is under the control of a TRE-regulated CMV minimal promoter, which drives expression of D56 in liver and muscle [15]. Expression of D56 in tilapia enhances resistance to $V$. vulnificus infection [15]. In addition, the D56 transgenic tilapia exhibit altered gut microbial profiles [15]. However, the underlying molecular mechanism involved in the resistance to $V$. vulnificus has not been studied using a transcriptomic approach.

We compared the liver transcriptomes between $V$. vulnificus-susceptible wild-type tilapia and D56 transgenic tilapia with enhanced resistance to the pathogen to reveal the particular genes responsible for the resistance $[15,16]$. The alterations in expression of key genes were identified by gene enrichment analysis with KEGG pathway and GO tools. We showed the involvement of fatty acid (FA)-associated genes and immunomodulatory genes in the development of resistance against $V$. vulnificus infection in tilapia.

\section{Results}

Expression of recombinant delta- 6 desaturase and delta-5 desaturase alters the transcriptome in tilapia

Wild-type and D56-transgenic tilapia were infected with $V$. vulnificus, and RNA was extracted from liver at 0, 6 and $24 \mathrm{~h}$ post-infection (hpi). Transcriptome sequencing of six groups of samples produced a total of 275,304,348 raw reads for wild-type and D56-transgenic tilapia. After filtering the data 48,315,226, 38,578,158 and 35,079,100 clean reads were obtained for wild-type tilapia fish at 0 ,
6 and $24 \mathrm{~h}$ infected samples, respectively, representing $92.12,89.24$ and $92.19 \%$ of raw reads (Table 1 ). Similarly, $37,449,898,49,652,212$ and 50,987,302 clean reads (90.62, 88.48 and $91.06 \%$ of raw reads) were obtained for D56-transgenic tilapia for 0, 6 and 24hpi samples, respectively (Table 1). A total of 42,622 unigenes were identified from the RNA-sequencing and filtered for two-fold change in expression between $V$. vulnificus challenged wild-type and D56-transgenic tilapia (Supplementary Figure S1). At the $0 \mathrm{~h}$ time-point, 3795 genes were upregulated and 1839 genes were downregulated in D56-transgenic tilapia (Fig. 1a). At $6 \mathrm{hpi}, 4365$ genes were upregulated and 1976 genes were downregulated (Fig. 1a). At 24 hpi, 4665 were upregulated and 2202 genes were downregulated (Fig. 1a). We could recognize the relevance of DEG between wild-type and D56transgenic tilapia at different time-points. We found that 1112 DEG existed at three time-points (Fig. 1b).

\section{Fatty acid-associated genes are altered in D56-transgenic} tilapia according to KEGG pathway analysis

Gene enrichment analysis using the KEGG pathway database showed a total of 24, 30 and 33 pathways were affected in D56-transgenic tilapia at 0, 6, and $24 \mathrm{hpi}$, respectively (Table 2, 34 ). Immediately after infection, altered expression of various FA-associated pathways, such as FA metabolism, FA elongation, FA biosynthesis, biosynthesis of unsaturated FA, and FA degradation were observed (Table 2). Differences in the FA degradation pathway were also observed between wild-type and D56-transgenic fish at $6 \mathrm{~h}$ post infection (Table 3 ), and the FA metabolism and FA degradation pathways were identified as differentially expressed at 24 hpi (Table 4).

\section{D56-transgenic tilapia exhibit altered immune-related gene expression in $\mathrm{GO}$ analysis}

The GO enrichment analysis showed a total of 28, 23 and 35 gene sets that were differentially expressed in

Table 1 Details of RNA sequence read

\begin{tabular}{llll}
\hline Sample & $\begin{array}{l}\text { Clean } \\
\text { Reads }\end{array}$ & $\begin{array}{l}\text { Filtered } \\
\text { Reads }\end{array}$ & $\begin{array}{l}\text { Mapping rate } \\
(\%)\end{array}$ \\
\hline WT-Liver-ctrl & $50,952,012$ & $48,315,226$ & $\mathbf{9 2 . 1 2}$ \\
WT-Liver-6h & $40,765,142$ & $38,578,158$ & $\mathbf{8 9 . 2 4}$ \\
WT-Liver-24h & $36,947,506$ & $35,079,100$ & $\mathbf{9 2 . 1 9}$ \\
D56-Liver-Ctrl & $39,667,224$ & $37,449,898$ & $\mathbf{9 0 . 6 2}$ \\
D56-Liver-6h & $52,706,028$ & $49,652,212$ & $\mathbf{8 8 . 4 8}$ \\
D56-Liver-24h & $54,266,436$ & $50,987,302$ & $\mathbf{9 1 . 0 6}$
\end{tabular}

RNA depletion of rRNA and organelle RNA was extracted from liver samples of wild-type and transgenic Tilapia fish expressing delta- 6 desaturase plus delta- 5 desaturase (D56) at 0,6 and $24 \mathrm{~h} \mathrm{~V}$. vulnificaus post infected conditions. The RNA was subjected to multiplexed RNA sequence. The total number of clean reads, filtered reads and RNA mapped reads for the six groups were shown in the table 


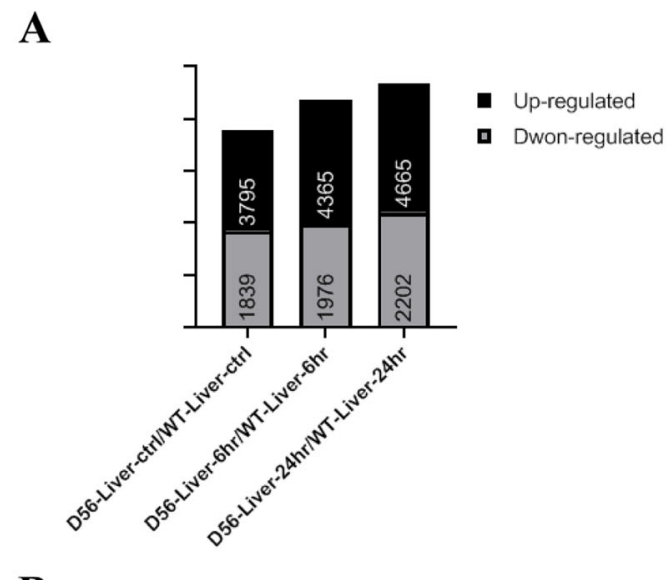

B

D56-Liver-ctrl/WT-Liver-ctrl

D56-Liver-6hr/WT-Liver-6hr

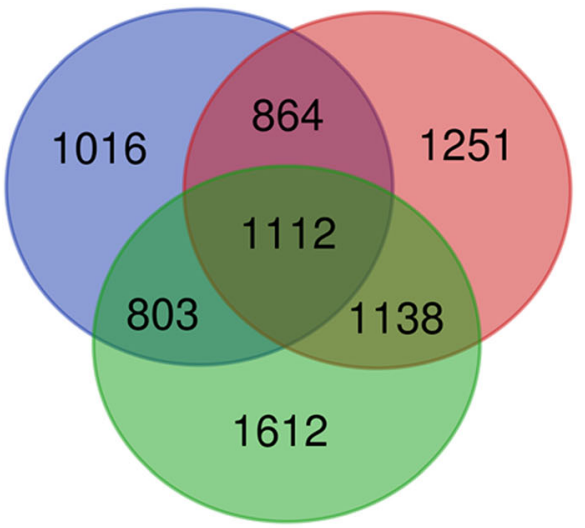

D56-Liver-24hr/WT-Liver-24hr

Fig. 1 Delta 5 and Delta6 transgenic (D56) tilapia fish alters the transcriptome profile. The comparative transcriptome data between wild-type / Delta 5 and Delta6 (D56) transgenic tilapia fish were mapped and differentially expressed genes were counted. a Differentially expressed genes with more than or equal to two fold change have been listed. Wild-type and D56 transgenic tilapia fish liver RNA were compared in 0, 6 and 24 $\mathrm{h}$ post infection with $V$. vulnificus. b Differentially expressed genes between wild-type and D56 transgenic tilapia fish at 0, 6 and $24 \mathrm{~h} \mathrm{~V}$. vulnificus post infected liver RNA. The number of the genes unique to specific infection condition and the number of genes commonly shared by two or three infection conditions are mentioned in the respective intersections

D56-transgenic and wild-type tilapia at 0,6 and $24 \mathrm{hpi}$ with $V$. vulnificus. Cellular component-associated GO terms, such as major histocompatibility (MHC) class II protein complexes and extracellular protein components, were altered at all the time-points examined (Table 5, 6, 7). Biological function-associated GO terms, such as defense response to bacterium, angiogenesis, immune response, antigen presenting and presentation and inflammatory response genes were also altered in D56transgenic tilapia. Altered molecular function-related GO terms included iron ion binding protein, cytokine and chemokine activity (Table 5, 6, 7). Taken together, the GO analysis revealed that inflammatory genes, chemokine-associated genes, cytokine-associated genes, immune-related genes and iron binding protein genes are differentially regulated in D56-transgenic and wildtype tilapia after $V$. vulnificus infection (Table 5, 6, 7).
We selected target genes with significant fold change, immune-related annotation and higher FPKM value for follow up research (Supplementary File 1).

\section{Ectopic D56 alters FA metabolism-related genes}

Since the KEGG analysis showed FA-associated pathways are altered in D56-transgenic tilapia, the expression of selected FA pathway-associated genes was analyzed by real-time PCR at $0,3,6,12,24$ and 48 hpi with $V$. vulnificus. Since the D56 transgenes (delta-6 desaturase and delta-5 desaturase) are associated with FA biosynthesis, we analyzed the expression pattern by $\mathrm{qPCR}$ at many time-points. Significant alterations in the expression of FA-associated genes were observed from the qPCR data (Fig. 2). Notably, ApoA4b was downregulated by D56transgenic tilapia at $24 \mathrm{hpi}$ (Fig. 2a). CPT1 was upregulated at 24 and 48 hpi (Fig. 2b), and PCK1 was 
Table 2 KEGG pathway enrichment analysis of wild-type and transgenic tilapia fish expressing delta-6 desaturase plus delta-5 desaturase (D56)

\begin{tabular}{|c|c|c|c|}
\hline Pathway ID & Pathway & DEGs with pathway annotation & Corrected $P$-value \\
\hline onl00620 & Pyruvate metabolism & 9 & $1 \mathrm{E}-07$ \\
\hline onl01212 & Fatty acid metabolism & 9 & $7 \mathrm{E}-06$ \\
\hline onl00010 & Glycolysis / Gluconeogenesis & 9 & $2 \mathrm{E}-05$ \\
\hline onl03320 & PPAR signaling pathway & 9 & $3 \mathrm{E}-05$ \\
\hline onl00071 & Fatty acid degradation & 7 & $3 \mathrm{E}-05$ \\
\hline onl00561 & Glycerolipid metabolism & 8 & $6 \mathrm{E}-05$ \\
\hline onl00062 & Fatty acid elongation & 6 & 0.0002 \\
\hline onl01040 & Biosynthesis of unsaturated fatty acids & 6 & 0.0003 \\
\hline onl00983 & Drug metabolism - other enzymes & 8 & 0.0005 \\
\hline onl00982 & Drug metabolism - cytochrome P450 & 6 & 0.0014 \\
\hline onl00564 & Glycerophospholipid metabolism & 8 & 0.0015 \\
\hline onl00980 & Metabolism of xenobiotics by cytochrome P450 & 6 & 0.0015 \\
\hline onl00480 & Glutathione metabolism & 7 & 0.0019 \\
\hline onl00410 & beta-Alanine metabolism & 4 & 0.0039 \\
\hline onl00020 & Citrate cycle (TCA cycle) & 4 & 0.0047 \\
\hline onl00330 & Arginine and proline metabolism & 5 & 0.0058 \\
\hline onl00061 & Fatty acid biosynthesis & 3 & 0.01 \\
\hline onl04910 & Insulin signaling pathway & 8 & 0.0133 \\
\hline onl00340 & Histidine metabolism & 3 & 0.0133 \\
\hline onl00280 & Valine, leucine and isoleucine degradation & 4 & 0.0208 \\
\hline onl04920 & Adipocytokine signaling pathway & 5 & 0.0257 \\
\hline onl00053 & Ascorbate and aldarate metabolism & 3 & 0.0326 \\
\hline onl00052 & Galactose metabolism & 3 & 0.0389 \\
\hline onl00270 & Cysteine and methionine metabolism & 4 & 0.0396 \\
\hline
\end{tabular}

upregulated at $24 \mathrm{hpi}$ (Fig. 2c). HNF4A was upregulated at $6 \mathrm{hpi}$ and downregulated at $24 \mathrm{hpi}$ (Fig. 2d). PPAR was upregulated at 6 and $12 \mathrm{hpi}$, but it was downregulated at $24 \mathrm{hpi}$ (Fig. 2e). These results showed that FA metabolism-related genes are altered in transgenic tilapia upon $V$. vulnificus infection.

\section{Ectopic D56 modulates immune response genes}

In addition to FA-associated genes, several inflammatory and immune responsive genes were altered in D56transgenic tilapia according to the $\mathrm{GO}$ enrichment analysis (Table $5,6,7)$. In addition, tilapia are known to express several antimicrobial peptides (AMPs), such as Tilapia Hepcidin, LEAP2, TP3, TP4, TP5 and Progranulin (PGRN), which have been reported to exert immunomodulatory functions. Hence, expression of genes associated with pro-inflammatory cytokines, immune responsive genes and AMPs were assessed at 0, 3, 6, 12, 24 and 48 hpi with $V$. vulnificus in wild-type and D56transgenic tilapia liver.

In D56-transgenic tilapia, the Complement $\mathrm{C} 1 \mathrm{q}$ subcomponent subunit $\mathrm{B}(\mathrm{Clqb})$ was upregulated at $6 \mathrm{hpi}$ and downregulated at $24 \mathrm{hpi}$. Complement factor $\mathrm{H}$ related protein 1 (CFHR1) was upregulated at 3, 6, 12, 24 and $48 \mathrm{hpi}$, and Complement factor D (CFD) was upregulated at $6 \mathrm{hpi}$ (Fig. 3). The AMPs also showed significant differences in expression between wild-type and D56-transgenic tilapia. Tilapia Hepcidin $(T H)$ was altered at 24 and $48 \mathrm{hpi}$; Binding protein I (BPI) was regulated at 24 and $48 \mathrm{hpi}$; liver-enriched antimicrobial peptide-2 (LEAP2) was altered at 12 and $24 \mathrm{hpi}$; Tilapia Piscidin $(T P) 3$ was differentially expressed at 6,24 , and $48 \mathrm{hpi}$;P4 was altered at $12 \mathrm{hpi}$; TP5 was altered at 6 hpi; PGRN expression differed at 0 and 48 hpi (Fig. 4). For the inflammatory factors, significant differences between wild-type and D56-transgenic tilapia were detected for $N F-\kappa B 2$ at 12 and $24 \mathrm{hpi} N F-\kappa B I$ was altered at $24 \mathrm{hpi}$; Toll-like receptor $(T L R)-2$ was altered at 12 hpi; TLR-5 was altered at 6, 24 and $48 \mathrm{hpi}$; Tumor necrosis factor $(T N F)-\alpha$ was altered at $12 \mathrm{hpi}$; Interleukin (IL)-1 $\beta$ was altered at $24 \mathrm{hpi}$ (Fig. 5). Other immunerelated genes were also affected. Peroxiredoxin $(P R D X) 1$ was altered at 6, 12 and $48 \mathrm{hpi}$; Atypical chemokine receptor $(A C K R) 4$ was altered at $48 \mathrm{hpi}$; Tissue inhibitor 
Table 3 KEGG pathway enrichment analysis of wild-type and transgenic tilapia fish expressing delta- 6 desaturase plus delta-5 desaturase (D56) infected with V. vulnificus for $6 \mathrm{~h}$

\begin{tabular}{|c|c|c|c|}
\hline Pathway ID & Pathway & DEGs with pathway annotation & Corrected $P$-value \\
\hline onl00983 & Drug metabolism - other enzymes & 10 & 7E-06 \\
\hline onl00330 & Arginine and proline metabolism & 8 & $1 \mathrm{E}-05$ \\
\hline onl00620 & Pyruvate metabolism & 6 & 0.0001 \\
\hline onl00561 & Glycerolipid metabolism & 7 & 0.0003 \\
\hline onl00380 & Tryptophan metabolism & 6 & 0.0003 \\
\hline onl00100 & Steroid biosynthesis & 4 & 0.0004 \\
\hline onl00500 & Starch and sucrose metabolism & 5 & 0.0006 \\
\hline onl00982 & Drug metabolism - cytochrome P450 & 6 & 0.001 \\
\hline onl00340 & Histidine metabolism & 4 & 0.0011 \\
\hline onl00480 & Glutathione metabolism & 7 & 0.0013 \\
\hline onl00071 & Fatty acid degradation & 5 & 0.0015 \\
\hline onl00010 & Glycolysis / Gluconeogenesis & 6 & 0.0029 \\
\hline onl00410 & beta-Alanine metabolism & 4 & 0.003 \\
\hline onl00053 & Ascorbate and aldarate metabolism & 4 & 0.004 \\
\hline onl01230 & Biosynthesis of amino acids & 6 & 0.0044 \\
\hline onl04145 & Phagosome & 11 & 0.006 \\
\hline onl00980 & Metabolism of xenobiotics by cytochrome P450 & 5 & 0.0061 \\
\hline onl00430 & Taurine and hypotaurine metabolism & 3 & 0.0082 \\
\hline onl00220 & Arginine biosynthesis & 3 & 0.0091 \\
\hline onl00260 & Glycine, serine and threonine metabolism & 4 & 0.0091 \\
\hline onl04060 & Cytokine-cytokine receptor interaction & 11 & 0.011 \\
\hline onl03060 & Protein export & 3 & 0.0121 \\
\hline onl01200 & Carbon metabolism & 7 & 0.0131 \\
\hline onl00280 & Valine, leucine and isoleucine degradation & 4 & 0.0165 \\
\hline onl04672 & Intestinal immune network for IgA production & 6 & 0.0199 \\
\hline onl04350 & TGF-beta signaling pathway & 6 & 0.027 \\
\hline onl00520 & Amino sugar and nucleotide sugar metabolism & 4 & 0.028 \\
\hline onl00270 & Cysteine and methionine metabolism & 4 & 0.0318 \\
\hline onl00310 & Lysine degradation & 4 & 0.0449 \\
\hline onl00360 & Phenylalanine metabolism & 2 & 0.0454 \\
\hline
\end{tabular}

of metalloproteinase (TIMP)2 was altered at $24 \mathrm{hpi}$ (Fig. 6).

\section{Ectopic D56 alters pro-inflammatory cytokines and CFD in whole blood sample}

We also measured gene expression in whole blood samples after challenge. Expression of cytokines, inflammatory factors and complement-related genes was analyzed by real-time qPCR. For inflammatory factors, cytokines and complement-related genes, we found that were several significant differences between wild-type and D56transgenic tilapia whole blood. TLR-5 was altered at 24 hpi; $N F-\kappa B 2$ was altered at $6,12,24$ and $48 \mathrm{hpi} ; N F-\kappa B I$ was altered at $0,6,12$, and $24 \mathrm{hpi} I L-1 \beta$ was altered at 24 and $48 \mathrm{hpi}$; $C 1 q b$ was altered at 0, 3 and $24 \mathrm{hpi}$; CFD was altered at $48 \mathrm{hpi}$ (Fig. 7a-f). In whole blood samples, the expression level of CPT1 was also different at 3 and 48 hpi (Fig. 7g). According to these results, protective and immune-related genes are induced in transgenic tilapia upon $V$. vulnificus infection.

\section{Discussion}

Tilapia (Oreochromis niloticus) is a staple product of the aquaculture industry, with annual consumption exceeding 3.7 million metric tons, as of 2014 [17]. Presently tilapia are grown in fresh-water pond culture systems in approximately 125 countries [18]. Breeding programs were used to develop improved versions of tilapia with high biomass [18]. Availability of whole genome sequence and RNA sequence data in recent years has 
Table 4 KEGG pathway enrichment analysis of wild-type and transgenic tilapia fish expressing delta- 6 desaturase plus delta-5 desaturase (D56) infected with V. vulnificus for $24 \mathrm{~h}$

\begin{tabular}{|c|c|c|c|}
\hline Pathway ID & Pathway & DEGs with pathway annotation & Corrected $P$-value \\
\hline onl00010 & Glycolysis / Gluconeogenesis & 17 & $2 \mathrm{E}-09$ \\
\hline onl01200 & Carbon metabolism & 17 & $1 \mathrm{E}-05$ \\
\hline onl04146 & Peroxisome & 13 & $3 \mathrm{E}-05$ \\
\hline onl00071 & Fatty acid degradation & 9 & $4 \mathrm{E}-05$ \\
\hline onl01230 & Biosynthesis of amino acids & 12 & $5 \mathrm{E}-05$ \\
\hline onl03320 & PPAR signaling pathway & 11 & 0.0002 \\
\hline onl00830 & Retinol metabolism & 9 & 0.0006 \\
\hline onl00051 & Fructose and mannose metabolism & 7 & 0.0007 \\
\hline onl00982 & Drug metabolism - cytochrome P450 & 8 & 0.0019 \\
\hline onl00980 & Metabolism of xenobiotics by cytochrome P450 & 8 & 0.0021 \\
\hline onl00590 & Arachidonic acid metabolism & 9 & 0.003 \\
\hline onl00640 & Propanoate metabolism & 5 & 0.0047 \\
\hline onl00100 & Steroid biosynthesis & 4 & 0.005 \\
\hline onl00330 & Arginine and proline metabolism & 7 & 0.0052 \\
\hline onl00410 & beta-Alanine metabolism & 5 & 0.0066 \\
\hline onl00650 & Butanoate metabolism & 4 & 0.0068 \\
\hline onl00053 & Ascorbate and aldarate metabolism & 5 & 0.0091 \\
\hline onl04060 & Cytokine-cytokine receptor interaction & 18 & 0.0101 \\
\hline onl00140 & Steroid hormone biosynthesis & 7 & 0.0108 \\
\hline onl00380 & Tryptophan metabolism & 6 & 0.011 \\
\hline onl00250 & Alanine, aspartate and glutamate metabolism & 6 & 0.0119 \\
\hline onl04920 & Adipocytokine signaling pathway & 8 & 0.012 \\
\hline onl00052 & Galactose metabolism & 5 & 0.0121 \\
\hline onl00350 & Tyrosine metabolism & 5 & 0.0144 \\
\hline onl04350 & TGF-beta signaling pathway & 10 & 0.0155 \\
\hline onl00620 & Pyruvate metabolism & 5 & 0.0185 \\
\hline onl04110 & Cell cycle & 10 & 0.0226 \\
\hline onl00260 & Glycine, serine and threonine metabolism & 5 & 0.0233 \\
\hline onl00360 & Phenylalanine metabolism & 3 & 0.0291 \\
\hline onl00760 & Nicotinate and nicotinamide metabolism & 5 & 0.0308 \\
\hline onl01212 & Fatty acid metabolism & 6 & 0.0369 \\
\hline onl02010 & $A B C$ transporters & 5 & 0.0396 \\
\hline onl00500 & Starch and sucrose metabolism & 4 & 0.0494 \\
\hline
\end{tabular}

allowed a greater understanding of the genetic makeup and expression profiles of different strains or groups of tilapia [19]. In fresh-water and brackish-water cultures, tilapia is prone to infection with the aquatic bacterial pathogen, V. vulnificus, which severely threatens the tilapia production $[4,18,20,21]$. $V$. vulnificus is a halophytic Gram-negative bacillus-type bacterium that can cause skin lesions, soft tissue dysfunction, and sepsisinduced mortality in tilapia or people who consume raw fish containing this pathogen $[22,23]$. The $V$. vulnificus strain 93 U204 has been isolated from an infected tilapia fish and its genome was previously sequenced [3].

Regulation of gene expression plays a major role in an organisms defense against pathogens [16]. Sequencingby-synthesis on an Illumina RNA-sequence platform has become a widely applied method for comparative transcriptome analysis [24, 25]. The primary sequence-bysynthesis data is contained in a multiplexed and mixed form with sequence information of all the groups in a $\mathrm{Bcl}$ file [26]. The complex $\mathrm{Bcl}$ form has to be converted 
Table 5 Gene ontology analysis of wild-type and transgenic tilapia fish expressing delta-6 desaturase plus delta-5 desaturase (D56)

\begin{tabular}{|c|c|c|c|}
\hline & Gene ontology term & Cluster frequency & Corrected $P$-value \\
\hline & Cellular component & & \\
\hline GO:0005576 & extracellular region & $12 / 392$ & 0.000548676 \\
\hline GO:0042613 & MHC class II protein complex & $3 / 392$ & 0.010994224 \\
\hline GO:0005882 & intermediate filament & $3 / 392$ & 0.011603118 \\
\hline \multirow[t]{2}{*}{ GO:0005887 } & integral component of plasma membrane & $4 / 392$ & 0.015772421 \\
\hline & Biological process & & \\
\hline GO:0006836 & neurotransmitter transport & $4 / 392$ & 0.001251906 \\
\hline GO:0043066 & negative regulation of apoptotic process & 2/392 & 0.007136566 \\
\hline GO:0009058 & biosynthetic process & $3 / 392$ & 0.009280905 \\
\hline GO:0006814 & sodium ion transport & $3 / 392$ & 0.010404294 \\
\hline GO:0001525 & angiogenesis & 2/392 & 0.010473526 \\
\hline GO:0019882 & antigen processing and presentation & $3 / 392$ & 0.012231075 \\
\hline GO:0007411 & axon guidance & 2/392 & 0.015786162 \\
\hline GO:0006811 & ion transport & $7 / 392$ & 0.021426441 \\
\hline GO:0006955 & immune response & $5 / 392$ & 0.027024892 \\
\hline GO:0007160 & cell-matrix adhesion & $2 / 392$ & 0.027243231 \\
\hline \multirow[t]{2}{*}{ GO:0006629 } & lipid metabolic process & 3/392 & 0.049135178 \\
\hline & Molecular function & & \\
\hline GO:0005179 & hormone activity & $5 / 392$ & 0.00069389 \\
\hline GO:0009055 & electron transfer activity & $3 / 392$ & 0.001715355 \\
\hline GO:0016491 & oxidoreductase activity & $7 / 392$ & 0.001873858 \\
\hline GO:0020037 & heme binding & $6 / 392$ & 0.004007088 \\
\hline GO:0019905 & syntaxin binding & $2 / 392$ & 0.004392611 \\
\hline GO:0016747 & $\begin{array}{l}\text { transferase activity, transferring acyl groups other } \\
\text { than amino-acyl groups }\end{array}$ & 2/392 & 0.006154289 \\
\hline GO:0016746 & transferase activity, transferring acyl groups & 3/392 & 0.006353286 \\
\hline GO:0005506 & iron ion binding & $5 / 392$ & 0.008148374 \\
\hline GO:0005216 & ion channel activity & $7 / 392$ & 0.00972373 \\
\hline GO:0004129 & cytochrome-c oxidase activity & 2/392 & 0.010473526 \\
\hline GO:0042626 & $\begin{array}{l}\text { ATPase activity, coupled to transmembrane } \\
\text { movement of substances }\end{array}$ & $3 / 392$ & 0.012878182 \\
\hline GO:0016705 & $\begin{array}{l}\text { oxidoreductase activity, acting on paired donors, } \\
\text { with incorporation or reduction of molecular oxygen }\end{array}$ & $4 / 392$ & 0.017329903 \\
\hline GO:0016702 & $\begin{array}{l}\text { oxidoreductase activity, acting on single donors } \\
\text { with incorporation of molecular oxygen, incorporation } \\
\text { of two atoms of oxygen }\end{array}$ & 2/392 & 0.030977671 \\
\hline
\end{tabular}

into a readable form, such as FASTQ format prior to further analysis [14]. The comprehensive computationbased resource, Gene ontology (GO), is extensively used to analyze the large amounts of FASTQ converted reads obtained from transcriptome analysis [27, 28]. Hence, we analyzed our transcriptome data with the KEGG pathway tool to search for major pathways altered in D56 transgenic tilapia compared to wild type. The major enriched genes in identified KEGG pathways and major genes or gene groups from GO analysis were measured by real-time PCR to confirm the regulation of gene expression by D56 transgenic tilapia fish compared to wild types.

Several reports have shown that resistance to infection can be enhanced in tilapia. AMPs, such as TP3 and TP4 have been reported to decrease the bacterial counts of $V$. vulnificus $[29,30]$. In addition, the multifunctional growth factor, PGRN, has been reported to modulate the immune response and improve survival of zebrafish infected with $V$. vulnificus [20]. Similarly, the granulin peptide, GRN-41, has been reported to exert antibacterial function against $V$. vulnificus [31]. When tilapia 
Table 6 Gene ontology analysis of wild-type and transgenic tilapia fish expressing delta-6 desaturase plus delta-5 desaturase (D56) infected with $V$. vulnificus for $6 \mathrm{~h}$

\begin{tabular}{|c|c|c|c|}
\hline GO number & Gene ontology term & Cluster frequency & Corrected $P$-value \\
\hline & Cellular component & & \\
\hline GO:0042613 & MHC class II protein complex & $4 / 388$ & 0.00111901 \\
\hline GO:0005576 & extracellular region & $10 / 388$ & 0.005273844 \\
\hline \multirow[t]{2}{*}{ GO:0000145 } & exocyst & $2 / 388$ & 0.023258951 \\
\hline & Biological process & & \\
\hline GO:0006879 & cellular iron ion homeostasis & $5 / 388$ & 7.19886E-06 \\
\hline GO:0019882 & antigen processing and presentation & $4 / 388$ & 0.001296795 \\
\hline GO:0006955 & immune response & $7 / 388$ & 0.00147042 \\
\hline GO:0005975 & carbohydrate metabolic process & $5 / 388$ & 0.006899818 \\
\hline GO:0006629 & lipid metabolic process & $4 / 388$ & 0.008996727 \\
\hline \multirow[t]{2}{*}{ GO:0006096 } & glycolytic process & $2 / 388$ & 0.026732255 \\
\hline & Molecular function & & \\
\hline GO:0004252 & serine-type endopeptidase activity & $12 / 388$ & $3.58836 \mathrm{E}-06$ \\
\hline GO:0004866 & endopeptidase inhibitor activity & $5 / 388$ & 7.56213E-05 \\
\hline GO:0016491 & oxidoreductase activity & $8 / 388$ & 0.00035261 \\
\hline GO:0003824 & catalytic activity & $8 / 388$ & 0.000514237 \\
\hline GO:0016788 & hydrolase activity, acting on ester bonds & $3 / 388$ & 0.000760589 \\
\hline GO:0004181 & metallocarboxypeptidase activity & $3 / 388$ & 0.001314446 \\
\hline GO:0004222 & metalloendopeptidase activity & $5 / 388$ & 0.005118182 \\
\hline GO:0016746 & transferase activity, transferring acyl groups & $3 / 388$ & 0.006176615 \\
\hline GO:0005125 & cytokine activity & $2 / 388$ & 0.009116399 \\
\hline GO:0008289 & lipid binding & $3 / 388$ & 0.032301052 \\
\hline GO:0005506 & iron ion binding & $4 / 388$ & 0.034540735 \\
\hline GO:0005509 & calcium ion binding & $9 / 388$ & 0.037632531 \\
\hline GO:0016616 & $\begin{array}{l}\text { oxidoreductase activity, acting on the } \mathrm{CH}-\mathrm{OH} \\
\text { group of donors, NAD or NADP as acceptor }\end{array}$ & $2 / 388$ & 0.040369571 \\
\hline GO:0004869 & cysteine-type endopeptidase inhibitor activity & $2 / 388$ & 0.042492616 \\
\hline
\end{tabular}

are fed with Epinecidin (Epi)-1-expressing transgenic Artemia, the mortality rate caused by $V$. vulnificus infection is decreased [32]. These studies demonstrate the utility of AMPs in controlling $V$. vulnificus infection.

D56-transgenic zebrafish and tilapia exhibit resistance to $V$. vulnificus infection $[5,15]$. The genes expressed in D56-transgenic fish, Atlantic salmon Fadsd5 and Fadsd6, play an important role in n-3 PUFA biosynthesis. Interestingly, exogenous FAs assimilated into the vibrio species affect the swimming motility, bacterial membrane structure, permeability, and virulence [33].

A comparative analysis of liver transcriptomes of wildtype and D56-transgenic tilapia infected with $V$. vulnificus was performed in this study. The multiplexed data of the six groups in the Bcl file were quality controlled, and sequence data for each group was multiplexed into FASTQ format. From the short reads, 88.48-92.19\% were mapped to the RNA genome in all samples. Thus, nearly $90 \%$ of the transcriptome was mapped for the comparative analysis. The KEGG pathway analysis showed several FA-associated pathways in wild-type and D56-transgenic tilapia groups at 0 hpi with $V$. vulnificus (Table 2). KEGG analysis also revealed that the drug metabolism pathway, amino acid pathways and Cytochrome 450 pathway were altered by D56 (Table 2). These results showed that the expression of D56 enhances FA biosynthesis, especially biosynthesis of unsaturated fatty acids, and it may also lead to the modulation of additional pathways. Several such pathways were also differentially activated between wild-type and D56-transgenic fish group $6 \mathrm{~h}$ and $24 \mathrm{~h}$ post infected with $V$. vulnificus (Table 3, 4). These data demonstrated the D56-mediated FA pathway may control several amino acid biosynthesis mechanisms in addition to stress response mechanisms related to pathogen resistance (Table 2, 3, 4).

The GO analysis showed that genes associated with inflammation, chemokine synthesis, iron homeostasis and immune response are altered by D56 expression in 
Table 7 Gene ontology analysis of wild-type and transgenic tilapia fish expressing delta-6 desaturase plus delta- 5 desaturase (D56) infected with V. vulnificus for $24 \mathrm{~h}$

\begin{tabular}{|c|c|c|c|}
\hline & Gene ontology term & Cluster frequency & Corrected $P$-value \\
\hline & Cellular component & & \\
\hline GO:0005576 & extracellular region & $20 / 614$ & 3.58031E-06 \\
\hline GO:0005737 & cytoplasm & $12 / 614$ & 4.79812E-05 \\
\hline GO:0042613 & MHC class II protein complex & $4 / 614$ & 0.005816676 \\
\hline GO:0005694 & chromosome & $2 / 614$ & 0.016811817 \\
\hline GO:0005667 & transcription factor complex & $2 / 614$ & 0.027216225 \\
\hline \multirow[t]{2}{*}{ GO:0005923 } & bicellular tight junction & $4 / 614$ & 0.038570306 \\
\hline & Biological process & & \\
\hline GO:0006096 & glycolytic process & $7 / 614$ & 1.13304E-07 \\
\hline GO:0006955 & immune response & $14 / 614$ & 4.69497E-07 \\
\hline GO:0006879 & cellular iron ion homeostasis & $6 / 614$ & $3.8588 \mathrm{E}-06$ \\
\hline GO:0019882 & antigen processing and presentation & $4 / 614$ & 0.006685535 \\
\hline GO:0007160 & cell-matrix adhesion & $3 / 614$ & 0.007455887 \\
\hline GO:0008610 & lipid biosynthetic process & $2 / 614$ & 0.01454755 \\
\hline GO:0042742 & defense response to bacterium & $2 / 614$ & 0.01454755 \\
\hline GO:0043066 & negative regulation of apoptotic process & $2 / 614$ & 0.016811817 \\
\hline GO:0009116 & nucleoside metabolic process & $2 / 614$ & 0.024421755 \\
\hline \multirow[t]{2}{*}{ GO:0006954 } & inflammatory response & $2 / 614$ & 0.033166717 \\
\hline & Molecular function & & \\
\hline GO:0005506 & iron ion binding & $27 / 614$ & 3.83798E-23 \\
\hline GO:0020037 & heme binding & $26 / 614$ & 1.05274E-19 \\
\hline GO:0016705 & $\begin{array}{l}\text { oxidoreductase activity, acting on paired donors, } \\
\text { with incorporation or reduction of molecular oxygen }\end{array}$ & $22 / 614$ & 2.62412E-19 \\
\hline GO:0016491 & oxidoreductase activity & $20 / 614$ & $5.06286 \mathrm{E}-12$ \\
\hline GO:0003824 & catalytic activity & $11 / 614$ & 0.000162737 \\
\hline GO:0004674 & protein serine/threonine kinase activity & $8 / 614$ & 0.000452088 \\
\hline GO:0004866 & endopeptidase inhibitor activity & $5 / 614$ & 0.000626808 \\
\hline GO:0008009 & chemokine activity & $6 / 614$ & 0.000757102 \\
\hline GO:0016702 & $\begin{array}{l}\text { oxidoreductase activity, acting on single donors with } \\
\text { incorporation of molecular oxygen, incorporation of } \\
\text { two atoms of oxygen }\end{array}$ & $4 / 614$ & 0.000867978 \\
\hline GO:0005125 & cytokine activity & $3 / 614$ & 0.001448524 \\
\hline GO:0042626 & $\begin{array}{l}\text { ATPase activity, coupled to transmembrane movement } \\
\text { of substances }\end{array}$ & $4 / 614$ & 0.00715045 \\
\hline GO:0008374 & O-acyltransferase activity & $2 / 614$ & 0.012426999 \\
\hline GO:0016887 & ATPase activity & $5 / 614$ & 0.013302119 \\
\hline GO:0016746 & transferase activity, transferring acyl groups & $3 / 614$ & 0.021148212 \\
\hline GO:0016773 & phosphotransferase activity, alcohol group as acceptor & $2 / 614$ & 0.021753335 \\
\hline GO:0016758 & transferase activity, transferring hexosyl groups & $3 / 614$ & 0.025460073 \\
\hline GO:0003779 & actin binding & $5 / 614$ & 0.029257274 \\
\hline GO:0004222 & metalloendopeptidase activity & $5 / 614$ & 0.03112367 \\
\hline GO:0019904 & protein domain specific binding & $2 / 614$ & 0.036314673 \\
\hline
\end{tabular}


A

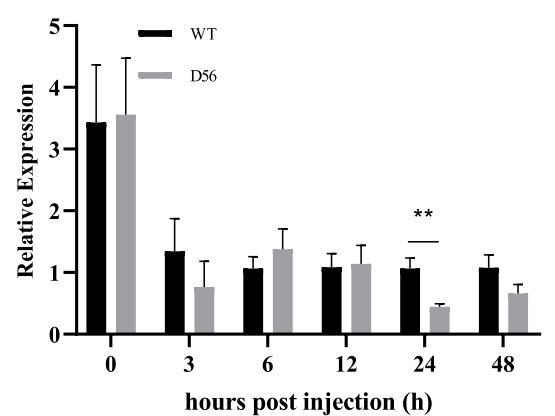

$\mathrm{C}$

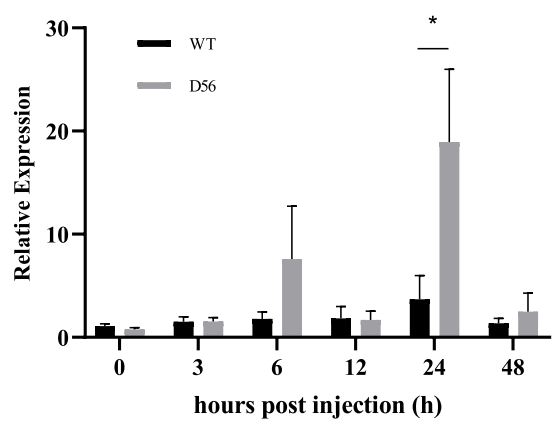

$\mathrm{E}$

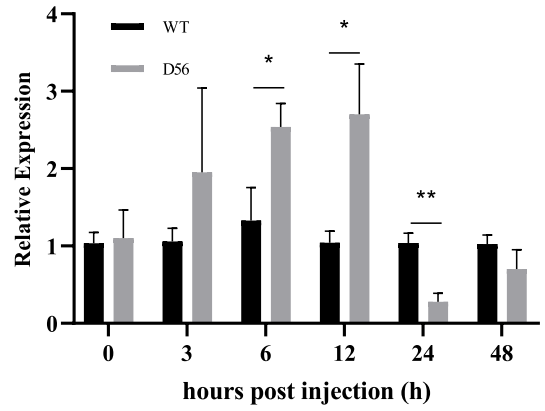

B

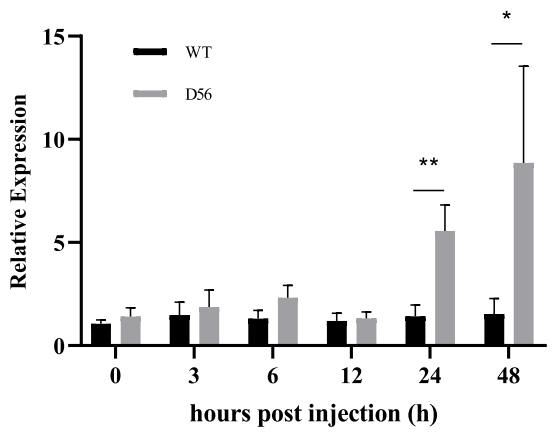

$\mathrm{D}$

HNF4A

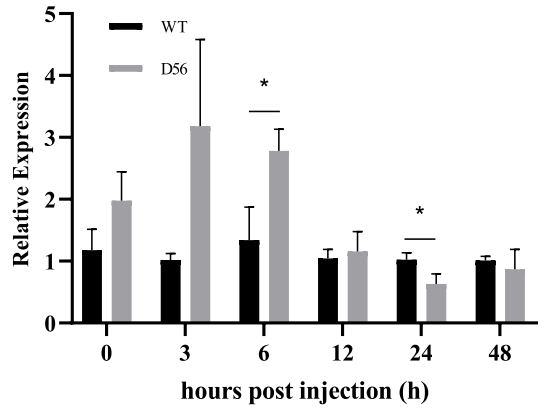

Fig. 2 Delta 5 and Delta 6 transgenic (D56) tilapia fish modulates the expression of Fatty acid associated gene expression. Wild-type and D56 transgenic tilapia fish challenged with $V$. vulnificus and liver samples from $0,3,6,12,24$ and $48 \mathrm{~h}$ post infected conditions were collected for qPCR. The fatty acid associated gene expression relative to EF-1a was estimated. a ApoA4b, b CPT1, c PCK1, d HNF4A, e PPARa. Values represented as Mean $\pm \operatorname{SEM}(n=5)$. Significance was determined by T-TEST $\left({ }^{*} P<0.05\right.$, ${ }^{* *} P<0.01$, ,** $\left.P<0.001\right)$

tilapia (Table 5). The iron binding genes are related to the AMP hepcidin [34-36]. Hence, it is possible that tilapia hepcidins (TH), such as TH1-5 and TH2-3, may be functionally affected by D56 expression. Previously, the anti- $V$. vulnificus activity of $\mathrm{TH} 2-3$ has been reported [37]. Thus, further investigations may explore the possibility that D56 regulates tilapia hepcidins. In addition, several AMPs that are secreted by tilapia fish are associated with innate immunity and immune modulatory functions. Hence, the FA-associated D56transgene expression may also directly alter the level of AMPs in tilapia fish. To address this possibility, the expression AMP-associated genes in tilapia was studied by
qPCR (Fig. 4). TH and BP1 were upregulated at $24 \mathrm{~h}$ and 48 hpi with $V$. vulnificus in D56-transgenic tilapia, suggesting the activation of these peptides was promoted by D56 to combat $V$. vulnificus infection (Fig. 4). The complement genes, $C 1 q b, C F H R 1$ and $C F D$ were also upregulated in D56-transgenic tilapia after $V$. vulnificus infection (Fig. 3). It is also possible that the plasma antibacterial function is stimulated by D56 expression in transgenic tilapia $[38,39]$. The inflammation-associated gene expression included early upregulation of TLR-2 and TNF- $\alpha$ in D56-transgenic fish (Fig. 5). The expression level of $N F-\kappa B$ did not vary, however, it is possible that the translocation of NF- $\mathrm{BB}$ from cytoplasm to 

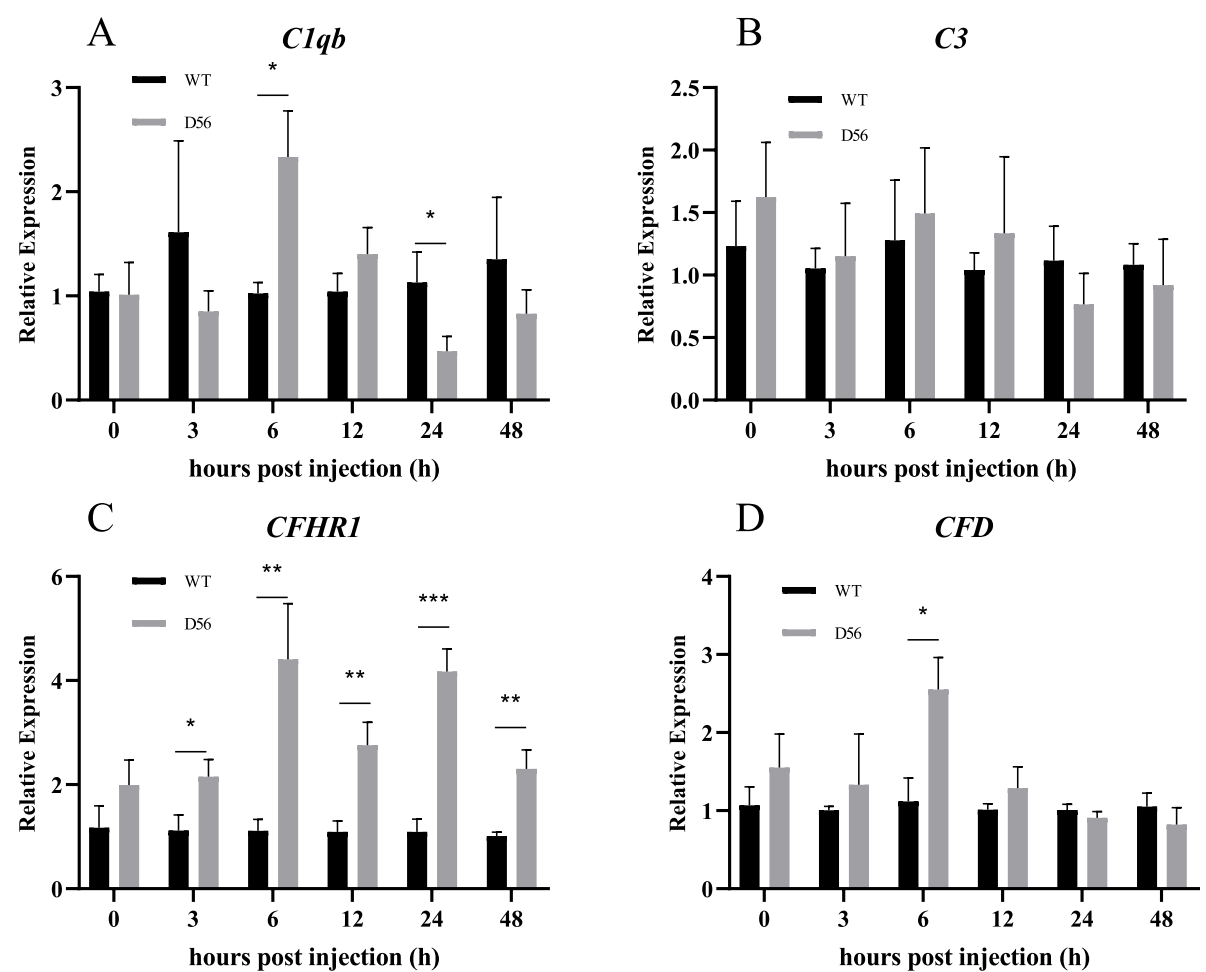

Fig. 3 Delta 5 and Delta 6 transgenic (D56) tilapia fish alters the Complements gene expression. Wild-type and D56 transgenic tilapia fish challenged with $V$. vulnificus and liver samples from $0,3,6,12,24$ and $48 \mathrm{~h}$ post infected conditions were collected for qPCR. The complements gene expression relative to EF-1a was estimated. a C19b, b C3, c CFHR1, d CFD. Error bar is Mean \pm SEM $(n=5)$. Significance was determined by T-TEST $\left({ }^{*} P<0.05,{ }^{* *} P<0.01,{ }^{* * *} P<0.001\right)$

nucleus triggered the activation of downstream genes, such as TNF- $\alpha$ [40]. The CD8-related Peroxiredoxin (PRDX)1, G-protein receptor associated Atypical Chemokine Receptor (ACKR)4, Tissue inhibitor of Metalloprotease (TIMP) 2 associated with extracellular matrix were also altered by the presence of D56 (Fig. 6). Probing the immunomodulatory gene expression in whole blood revealed that genes such as NF- $K B 2, T L R-5, I L-1 \beta$, $C F D$ and $C 1 q b$ were upregulated by D56 after $V$. vulnificus infection (Fig. 7). Altogether, the FA-associated pathways triggered in the D56-transgenic tilapia appear to regulate a variety of immune-related genes that may serve to enhance resistance to $V$. vulnificus infection.

\section{Conclusions}

We compare the results between NGS and qPCR (Supplementary Table S2). We found that TLR-2, TLR-5 and $A C K R 4$ were induced in the D56-transgenic line. TLR-5 and TLR-2 may stimulate a pro-inflammatory response, and ACKR4 blocks chemokine signaling. Through the regulation of CFHR1 and CFD, n-3 PUFAs can modulate the complement system. Moreover, AMP-associated genes, including TP3, TP4, TP5, TH, PGRN, BPI and LEAP2, may possibly be regulated by $\mathrm{n}-3$ PUFAs (Fig. 8a). In terms of downstream effects, HNF4A and PPARs may induce $C P T 1$ and PCK1. This action would enhance ATP production from FA oxidation and help the host overcome infection by pathogenic bacteria (Fig. $8 b$ ). Furthermore, $N F-\kappa B$ downstream genes, such as TNF- $\alpha$ and $I L-1 \beta$, may suppress pathogens (Fig. 8c). In this study, we measured the transcriptome-wide responses of wildtype and D56-transgenic tilapia to $V$. vulnificus infection. Several pathways associated with inflammation, immune response, chemokine and cytochrome were specifically altered in D56-transgenic tilapia, as revealed by KEGG pathway and GO analysis of the wild-type and D56transgenic tilapia at the baseline $(0 \mathrm{hpi})$ and after infection (6 and $24 \mathrm{hpi}$ ). These results suggest that D56 may modulate pro-inflammatory cytokines, AMPs and immune response genes to enhance resistance to $V$. vulnificus (Fig. 8).

\section{Methods}

Tilapia fish and culture

Tilapia fish Oreochromis niloticus were acclimatized in FRP tanks with $2000 \mathrm{l}$ capacity under the controlled conditions of $28{ }^{\circ} \mathrm{C}$ with a $13 \mathrm{~h}$ light/ $11 \mathrm{~h}$ dark cycle with Alanine rich food at $4 \%$ body weight per day for 45 days. Wild-type and transgenic tilapia fish with dual expression of delta- 6 desaturase in liver and delta- 5 desaturase 

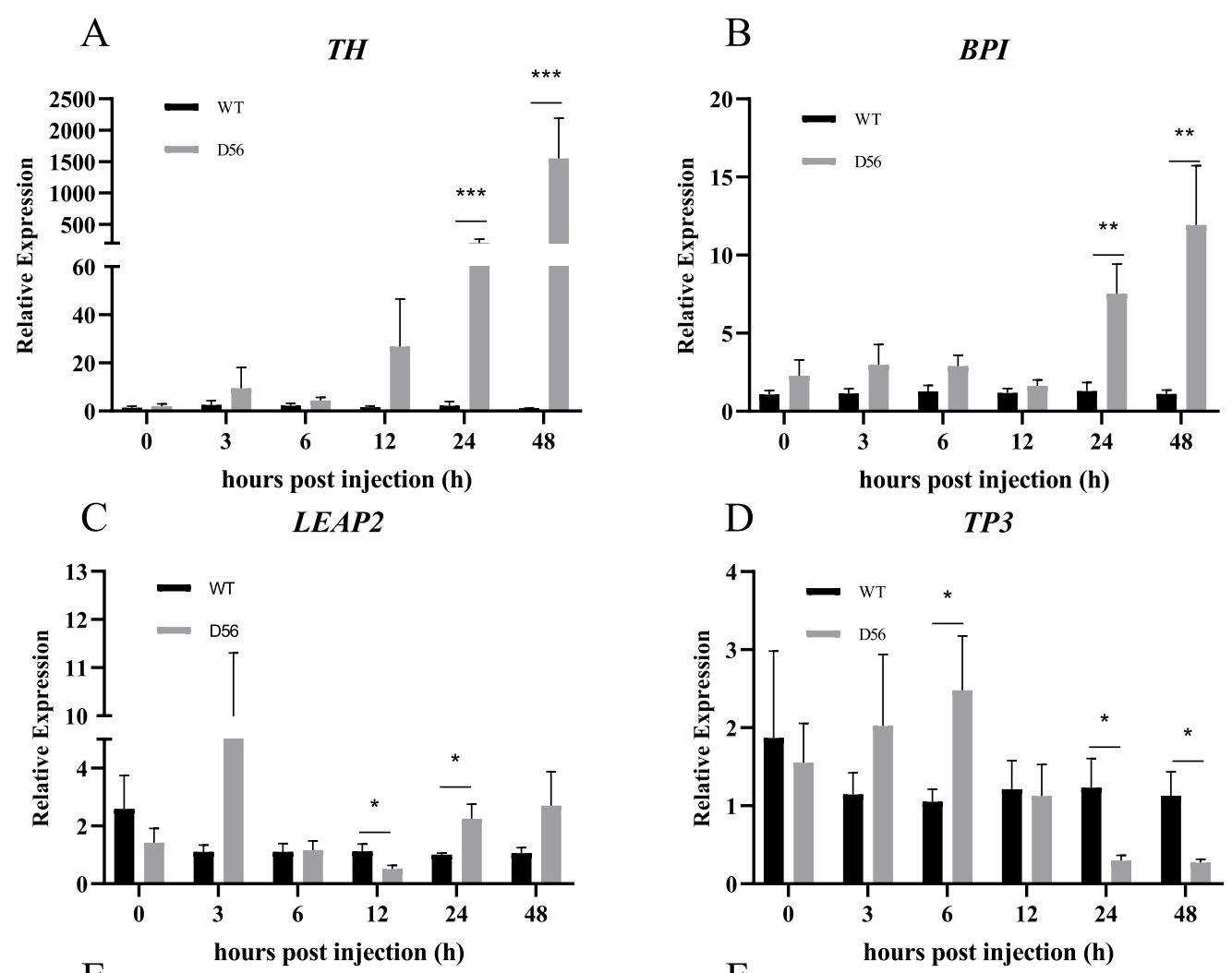

E

TP4
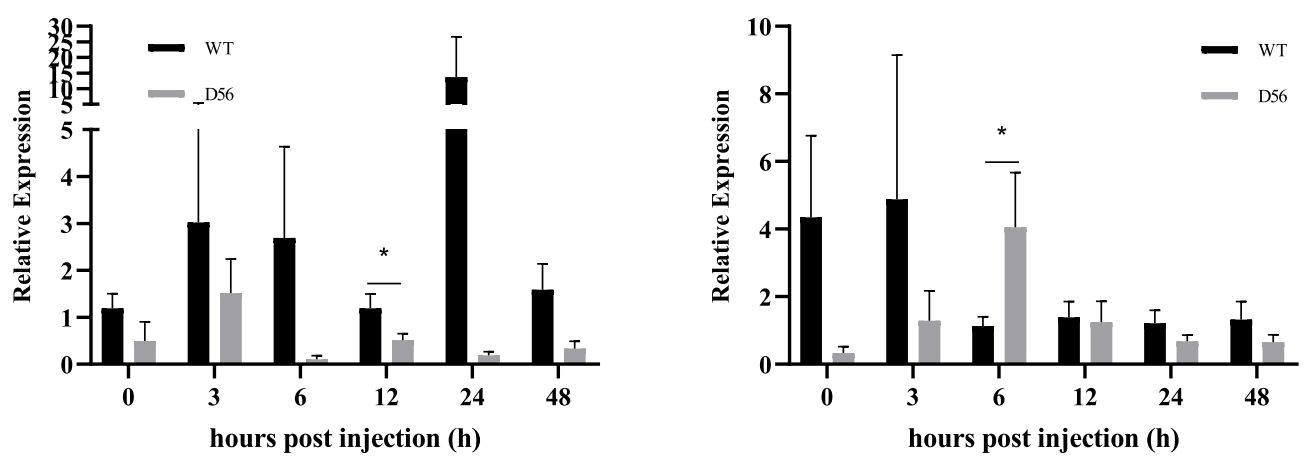

G

PGRN

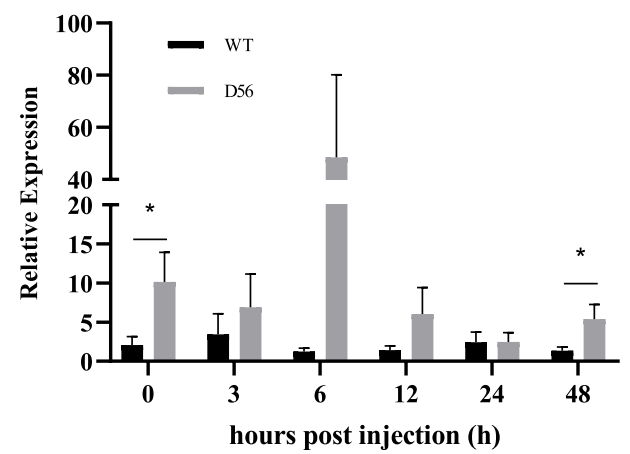

Fig. 4 (See legend on next page.) 
(See figure on previous page.)

Fig. 4 Delta 5 and Delta 6 transgenic (D56) tilapia fish alters the Antimicrobial peptide (AMP) specific gene expression. Wild-type and D56 transgenic tilapia fish challenged with $V$. vulnificus and liver samples from $0,3,6,12,24$ and $48 \mathrm{~h}$ post infected conditions were collected for qPCR. The AMP specific gene expression relative to EF-1a was estimated. a TH, b BP1, c LEAP2, d TP3, e TP4, f TP5, g PGRN. Values represented as Mean $\pm \operatorname{SEM}(n=5)$. Significance was determined by T-TEST $\left({ }^{*} P<0.05,{ }^{* *} P<0.01,{ }^{* *} P<0.001\right)$

in muscle (D56) were gift from Dr. Jen-Leih Wu's lab from Institute of Cellular and Organismic Biology, Academia Sinica, Taipei, Taiwan [15]. After 45 days the wild-type tilapias reached an average of $8.93 \pm 0.98 \mathrm{~cm}$ length and $11.52 \pm 3.55 \mathrm{~g}$ weight. The transgenic tilapias were $10.06 \pm 1.02 \mathrm{~cm}$ in length and $16.25 \pm 3.61 \mathrm{~g}$ weight. The experimental fish were handled after getting approval from the Institutional Animal Care and Use Committee (IACUC) at Academia Sinica, Taiwan, and the IACUC guidelines were followed. According to the formula: $\mathrm{n}=\left(\mathrm{Z} \_1\right.$-beta ${ }^{*} \operatorname{sqrt}(\mathrm{p} 1 \mathrm{q} 1+\mathrm{p} 2 \mathrm{q} 2)+\mathrm{Z} \_1$-alpha/2 ${ }^{*} \operatorname{sqrt}\left(2 *\right.$ p_avg $* \mathrm{q}_{-}$avg $\left.)\right)^{\wedge} 2 / \operatorname{delta}^{\wedge} 2, \mathrm{p} 2=\mathrm{p} 1+$ delta, $\mathrm{p}_{-}$ avg $=(\mathrm{p} 1+\mathrm{p} 2) / 2$, q_avg $=1$ - p_avg. The minimum number of samples in each group was $5(K=2, \alpha=0.05$,
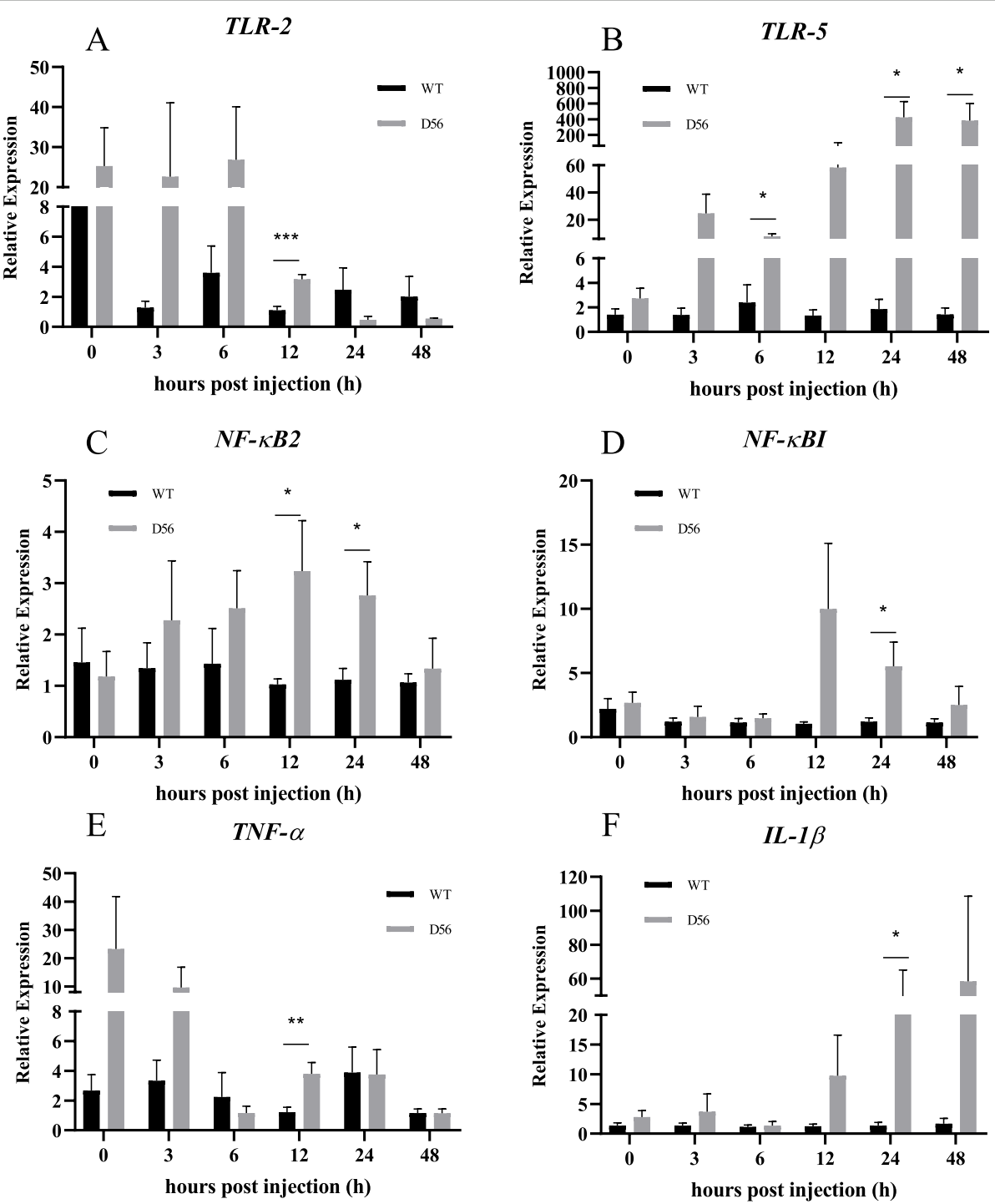

Fig. 5 Delta 5 and Delta 6 transgenic (D56) tilapia fish modulates the pro-inflammatory cytokine gene expression. Wild-type and D56 transgenic tilapia fish challenged with $V$. vulnificus and liver samples from $0,3,6,12,24$ and $48 \mathrm{~h}$ post infected conditions were collected for qPCR. Representative pro-inflammatory cytokine gene expression relative to EF-1a was estimated. a $T L R-2, \mathbf{b} T L R-5, \mathbf{c} N F-K B 2, \mathbf{d} N F-K B I$, e $T N F-a$, f $I L-1 \beta$. Values represented as Mean \pm SEM $(n=5)$. Significance was determined by T-TEST $\left({ }^{*} P<0.05,{ }^{* *} P<0.01, * * P<0.001\right)$ 


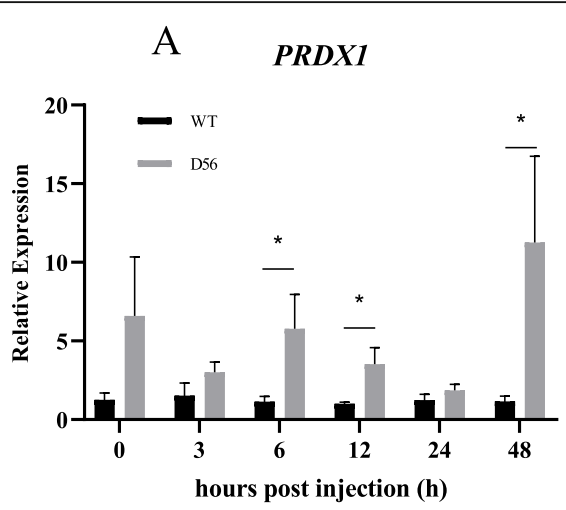

\section{B}

\section{ACKR4}
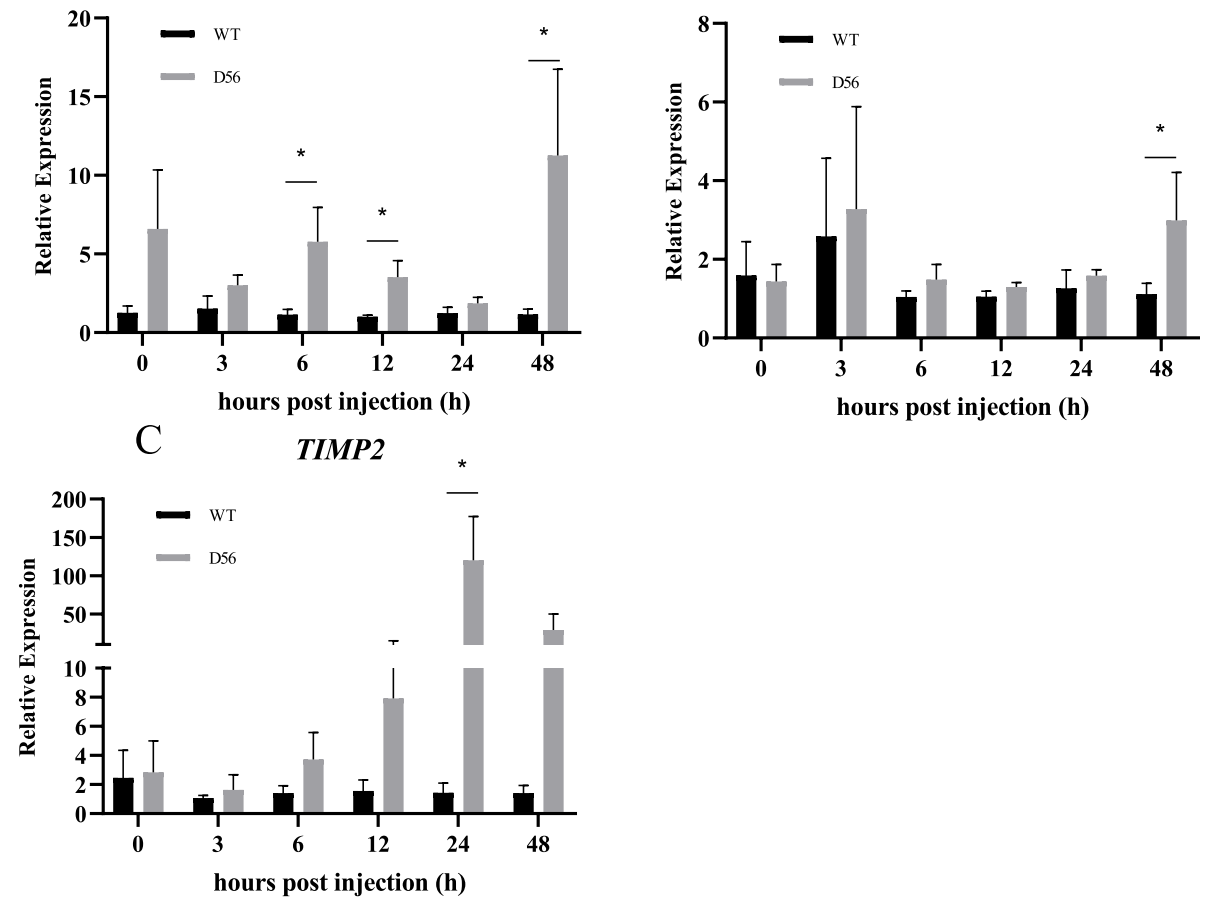

Fig. 6 Delta 5 and Delta 6 transgenic (D56) tilapia fish alters PRDX1, ACRK4 and TIMP2 gene expression. Wild-type and D56 transgenic tilapia fish challenged with $V$. vulnificus and liver samples from $0,3,6,12,24$ and $48 \mathrm{~h}$ post infected conditions were collected for qPCR. Expression of a PRDX1, b ACKR4, c TIMP2 genes relative to EF-1a was estimated. Values represented as Mean \pm SEM $(\mathrm{n}=5)$. Significance was determined by T-TEST $\left({ }^{*} P<0.05\right.$, ${ }^{* *} P<0.01$, $\left.{ }^{* *} P<0.001\right)$

$1-\beta=1-0.2=0.8, \mathrm{p} 1=37 \%$ and $\Delta=74 \%)$. A total of 66 animals were used in this study (5 samples * 2 groups * 6 time-points $=60$ for qPCR analysis; 6 animals were used for transcriptome analysis). The method for euthanasia was rapid chilling by soaking the animal in ice for $10 \mathrm{~min}$. The ratio of ice to water was roughly 5:1. Ice and water were both measured volumetrically for a total volume of roughly $10 \mathrm{~L}$ [41].

\section{Expression of D56 in transgenic Tilapia}

The vector construct and expression of D56 has been explained previously [15]. In brief the delta- 6 desaturase transgenic line driven by muscle-specific $C K M b$ promoter express the Fadsd6 with TcFP11 reporter was created by co-injecting the plasmid into the embryo. Similarly delta- 5 desaturase transgenic line driven by liver-specific Fabp10 promoter to express the Fadsd5 with TcFP13 reporter was created separately. The delta 5 and delta 6 transgenic tilapia have been crossed and the offspring carrying D56 has been selected for the experiment. Atlantic salmon (Salmon salar) delta-6 desaturase gene Fadsd6 and delta-5 desaturase gene Fadsd5 were used in the construct.

\section{V. vulnificus culture and infection in to tilapia}

The pathogen $V$. vulnificus strain 93 U204 was initially cultured in a TCSB (Thiosulfate Citrate Bile Salts Sucrose, BD Difco $\left.{ }^{\mathrm{rm}}\right)$ agar plate for $16 \mathrm{~h}$ at $28^{\circ} \mathrm{C}$ [3]. From overnight cultured plate a single colony was picked and cultured in $3 \mathrm{ml}$ TSB (Tryptone Soy Broth, BD Difco ${ }^{\mathrm{mm}}$ ) with $1.5 \% \mathrm{NaCl}$ in an incubated shaker at $28^{\circ} \mathrm{C}$ with $200 \mathrm{rpm}$ up to four hours until the $\mathrm{OD}_{600 \mathrm{~mm}}$ reached 0.7 to 0.9. At the end of acclimatization the wild-type and transgenic fish were subjected to $V$. vulnificus challenge by i.p. injecting $50 \mu \mathrm{l}$ of diluted TSB containing $1.9 \times$ $10^{2}$ CFU of $V$. vulnificus for each fish. Thirty fishes in each group was infected with $V$. vulnificus and five fish per each group was sacrificed at $0,3,6,12,24$ and $48 \mathrm{~h}$ post infection for molecular analysis.

\section{Extraction of total RNA}

The fish was sacrificed at the end of each time point of the experiment and liver tissue were collected for total RNA extraction. The liver tissue was homogenized and total RNA was extracted using Trizol ${ }^{\circ}$ Reagent by following the company protocol (Invitrogen, USA). Purified RNA was quantified at OD260nm using a ND-1000 spectrophotometer (NanodropTechnology, USA) and 

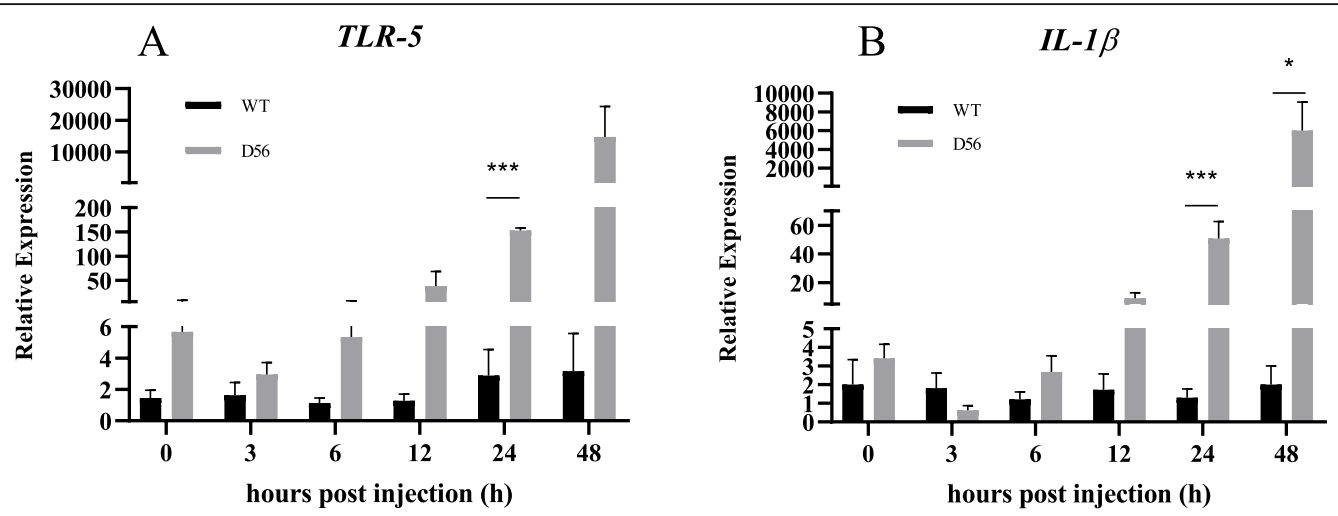

C

$N F-\kappa B 2$

D

$N F-\kappa B I$
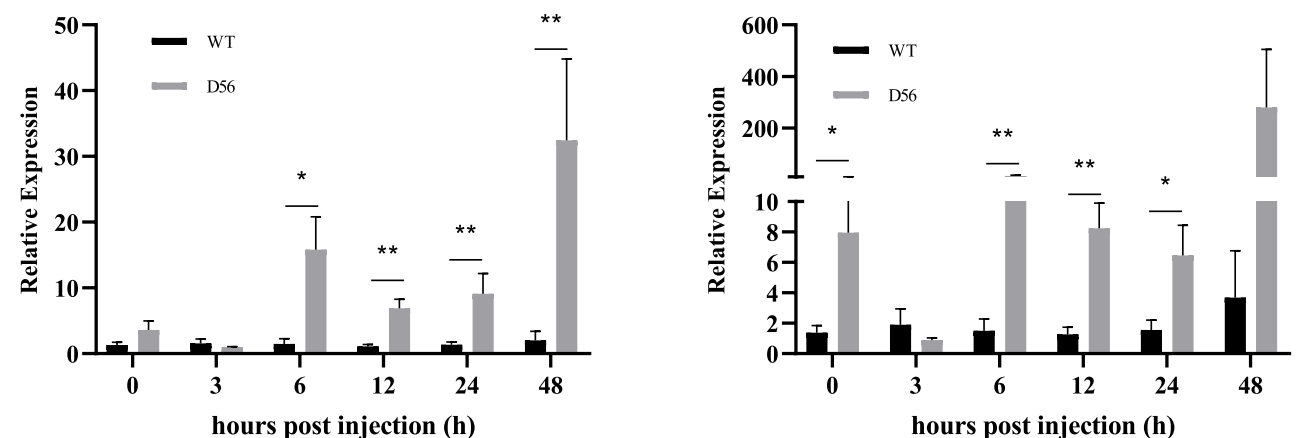

E

CFD

F

$$
C 1 q b
$$
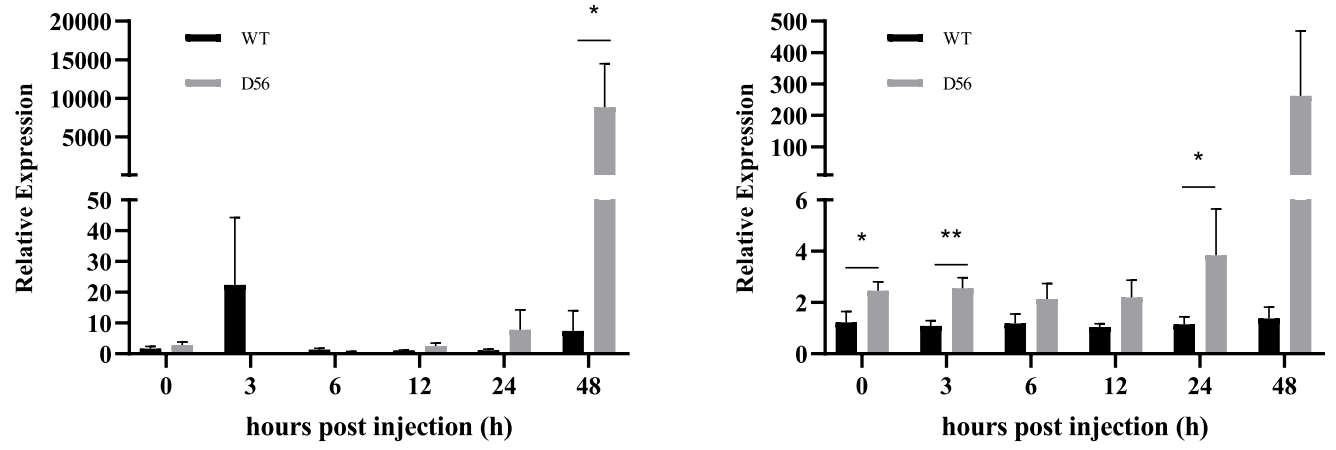

G

CPT1

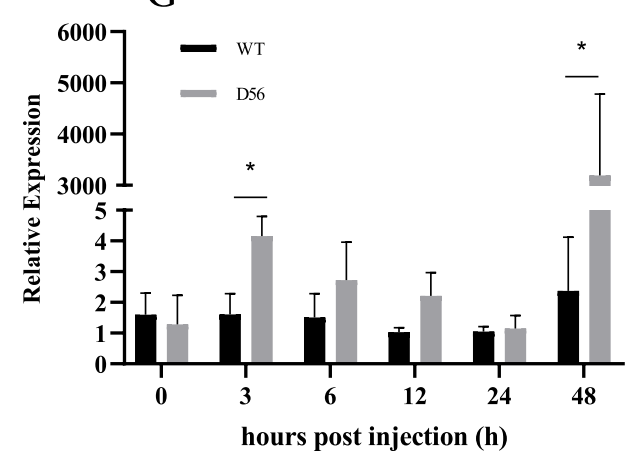

Fig. 7 (See legend on next page.) 
(See figure on previous page.)

Fig. 7 Delta 5 and Delta 6 transgenic (D56) tilapia fish modulates the pro-inflammatory cytokines and immune-related genes in whole blood. Wild-type and D56 transgenic tilapia fish challenged with $V$. vulnificus and whole blood samples from 0, 3, 6, 12, 24 and $48 \mathrm{~h}$ post infected

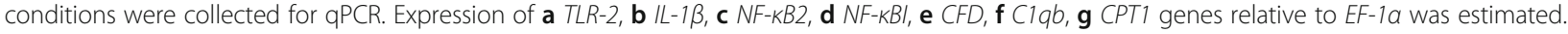
Values represented as Mean \pm SEM $(n=5)$. Significance was determined by T-TEST $\left({ }^{*} P<0.05,{ }^{* *} P<0.01,{ }^{* * *} P<0.001\right)$

qualitated by using a Bioanalyzer 2100 (Agilent Technology, USA) with RNA 6000 LabChip kit (Agilent Technology, USA).

\section{RNA library construction and transcriptome analysis}

RNA extracted from three fish in each group was used for the RNA library construction. Illumina'sTruSeq Stranded Total RNA Library Prep Gold Kit (Cat. 20,020, 598) was used for library construction. AMPure XP beads (Beckman Coulter, USA) kit was used for size selection. The sequence was determined using Illumina's sequencing-by-synthesis (SBS) technology (Illumina, USA) (150 bp, pair-end, 6Gb). Bcl2fastq v2.20 (Illumina, USA) software was used to trim adapter sequence, eliminating Unique Molecular Identifier (UMI) sequences and conversion of BCL files to the per-read FASTQ format. Both adaptor clipping and sequence quality trimming were performed using Trimmomatic v0.36 with a sliding-window approach. After FASTQC, raw reads were aligned to the downloaded tilapia reference genome using HISAT2 software. Differential expression analysis was performed using cuffdiff (cufflinks v2.2.1) with genome bias detection/correction and Welgene Biotech's inhouse pipeline (Supplementary Figure S1). Functional enrichment assay in differentially expressed genes of each experiment design was performed using clusterProfiler v3.6. Genes with low expression level $(<0.3$ FPKM value) in either or both of the treated and control samples were excluded (Supplementary File S1). Genes with $p$ value $\leq 0.05$ and $\geq 2$-fold changes were considered significantly differentially expressed.

\section{Gene ontology (GO) and Kyoto encyclopedia of genes and genomes (KEGG) analysis}

$\mathrm{GO}$ enrichment analysis of the differentially expressed genes (DEGs) was implemented using the clusterProfiler package. Pathway enrichment was used for KEGG analysis (https://www.genome.jp/kegg/), which uses public databases to examine Oreochromis niloticus biological pathways. The datasets generated and analyzed during

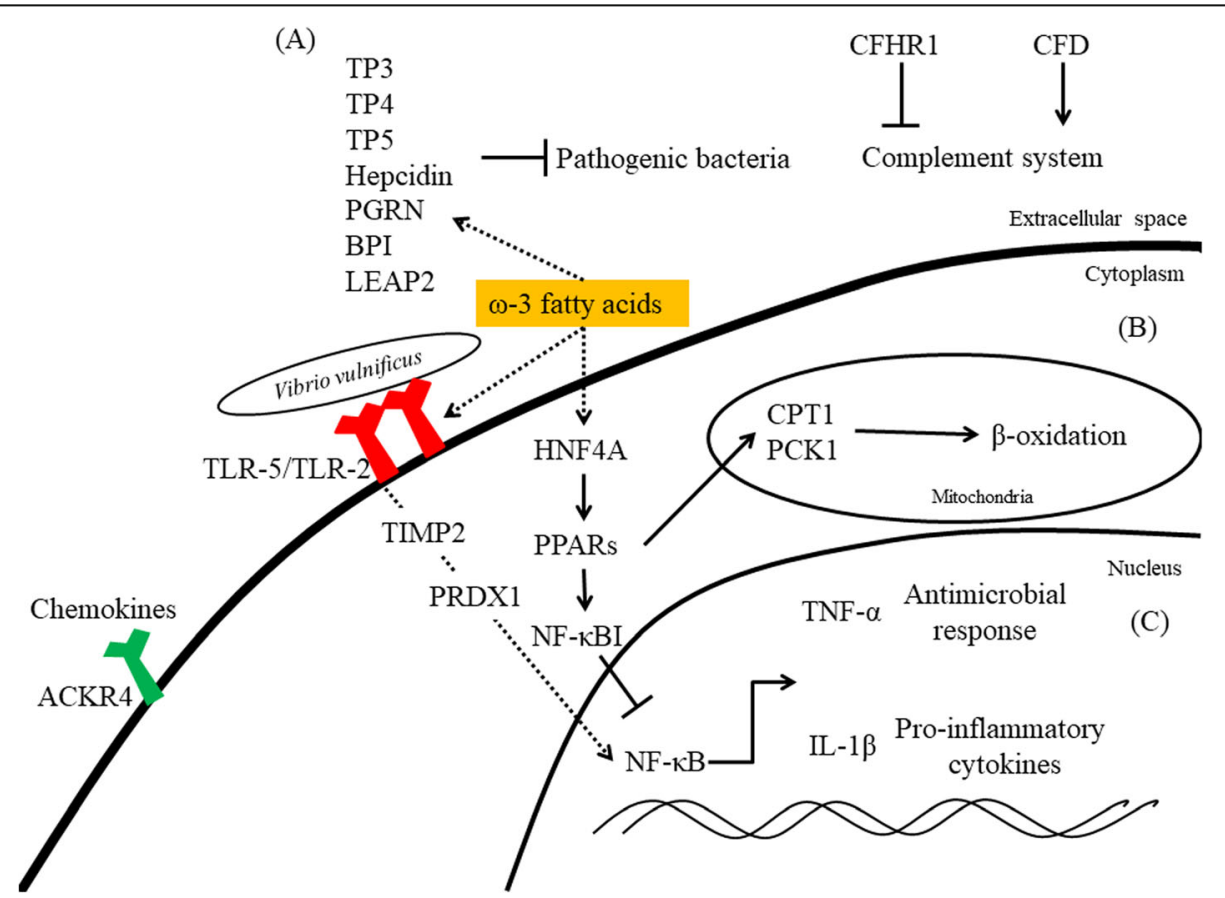

Fig. 8 Proposed mechanism of Delta 5 and Delta 6 transgenic (D56) mediated $\omega-3$ fatty acid metabolism to enhance resistance against $V$. vulnificus in Tilapia. a The $\omega-3$ fatty acids synthesized in D56 transgenic fish regulates or activates the extra cellular acting agents such as Antimicrobial peptide (AMP), Complement system and ACKR4 by altering their expression. $\mathbf{b}$ In cytosol D56 mediated $\omega$-3 fatty acid regulates HNF4A, TLR-5 and TLR-2, PPARs, TIMP2 and PRDX1. c In nucleus transcription factors such as NF-KB is altered to regulate the proinflammatory cytokines. (Image from ourselves) 
the current study are available in the NCBI repository, https://www.ncbi.nlm.nih.gov/bioproject/705417 (Accession: PRJNA705417 ID: 705417) (Accession: PRJNA705417 ID: 705417).

\section{Gene expression analysis with qPCR} cDNA was obtained from $0.5 \mu \mathrm{g}$ of total RNA using ReverTra Ace ${ }^{\circ}$ qPCR RT Master Mix with gDNA Remover kit (TOYOBO, Japan). qPCR was performed using Applied BiosystemsStepOnePlus ${ }^{\text {Tw }}$ System (ABI, USA) machine. The qPCR reaction mix comprise of $5 \mu$ l SYBR $^{\circ}$ Green Realtime PCR Master Mix (TOYOBO, Japan), $0.5 \mu \mathrm{l}(10 \mu \mathrm{M})$ each of gene specific primers (Supplementary Table S1), and $4 \mu \mathrm{l}$ of twenty times diluted cDNA. Amplification was performed with an initial $95^{\circ} \mathrm{C}$ for $1 \mathrm{~min}$ and 40 cycles of $95^{\circ} \mathrm{C}$ for $15 \mathrm{~s}, 60^{\circ} \mathrm{C}$ for $15 \mathrm{~s}$ and $72^{\circ} \mathrm{C}$ for $45 \mathrm{~s}$ (Supplementary Figure S2). Gene expression relative to $E F-1 \alpha$ was estimated by $2_{\mathrm{T}}{ }^{-\Delta \Delta C}$ method as described [42].

\section{Statistical analysis}

Data are presented as the mean \pm standard error of mean (SEM). Statistical analysis was performed using Student's $t$-TEST $\left(* P<0.05,{ }^{* *} P<0.01\right.$, $\left.{ }^{* * * *} P<0.001\right)$.

\section{Abbreviations}

ACKR4: Atypical chemokine receptor 4; ApoA4b: Apolipoprotein A-IV-b; BPI: Bactericidal permeability-increasing protein; CFD: Complement factor D; CFHR1: Complement factor H-related protein 1; CPT1: Carnitine OPalmitoyltransferase 1; HNF4A: Hepatocyte nuclear factor 4, Alpha; IL1及: Interleukin 1 beta; LEAP2: Liver-expressed antimicrobial peptide 2; NFkB2: Nuclear factor kappa B subunit 2; NFkBI: NF-kappa-B inhibitor alpha; TH: Tilapia hepcidin; TIMP2: Tissue inhibitor of metalloproteinases 2; TLR2: Toll- like receptor 2; TLR-5: Toll- like receptor 5; TP: Tilapia piscidin; TNFa: Tumor necrosis factor alpha; PCK1: Phosphoenolpyruvate carboxykinase 1 PGRN: Progranulin; PPARa: Peroxisome proliferator activated receptor alpha; PRDX1: Peroxiredoxin-1

\section{Supplementary Information}

The online version contains supplementary material available at https://doi. org/10.1186/s12864-021-07521-5

\section{Additional file 1: Supplementary Figure S1. Analysis of sample correlation.}

Additional file 2: Supplementary Figure S2. Melting curve analysis of target genes.

Additional file 3: Supplementary Table S1. List of primers used in QPCR.

Additional file 4: Supplementary Table S2. Comparison of RNA-seq and GRT-PCR.

Additional file 5: Supplementary File S1. DEG between D56 and WT.

\section{Acknowledgements}

We would like to thank Dr. Marcus J. Calkins (ICOB, Academia Sinica) for English language editing. We are grateful thank the aquaculture groups of the Marine Research Station, Institute of Cellular and Organismic Biology, Academia Sinica for their help with the tilapia maintenance.

\section{Authors' contributions}

P.Y.T. performed experiment and analyzed the data. P.Y.T., S.J.H., J.L.W. and J.Y.C. designed the study. P.Y.T., V. R. and J.Y.C. participated in drafting and revision of the manuscript. All authors have read and approved the final manuscript, and ensure that this is the case.

\section{Funding}

Research funding for this study was partly from the academic committee members of Innovative Translational Agricultural Research Program (AS-109ITAR-L081) (Academia Sinica) to Dr. Jyh-Yih Chen. The funders had no role in study design, data collection and analysis, decision to publish, or preparation of the manuscript. This work was financially supported by the iEGG and Animal Biotechnology Center from the Feature Areas Research Center Program within the framework of the Higher Education Sprout Project by the Ministry of Education (MOE-109-S-0023-A) in Taiwan. This work was partly financially supported by the 109-2313-B-001-007-MY3, 109-2622-B-001-002CC1, or 108-2313-B-001-006-from the Ministry of Science and Technology (Taiwan). The funders played no part in study design, data collection and analysis, decision to publish, or preparation of the manuscript.

\section{Availability of data and materials}

The datasets generated and analyzed during the current study are available in the NCBI repository, https://www.ncbi.nlm.nih.gov/bioproject/705417 (Accession: PRJNA705417 ID: 705417 ).

\section{Declarations}

Ethics approval and consent to participate

The study received approval from the institutional review board of Academia Sinica.

Consent for publication

Not Applicable.

\section{Competing interests}

The authors declare that they have no competing financial interests.

\section{Author details}

${ }^{1}$ Marine Research Station, Institute of Cellular and Organismic Biology, Academia Sinica, 23-10 Dahuen Rd., Jiaushi, llan 262, Taiwan. ${ }^{2}$ Institute of Cellular and Organismic Biology, Academia Sinica, 128 Academia Road, Section 2, Nankang, Taipei 115, Taiwan. ${ }^{3}$ Department of Plant Breeding 7 Genetics, Anbil Dharmalingam Agricultural College \& Research Institute, Tamil Nadu Agricultural University, Tiruchirapalli, Tamil Nadu 620027, India. ${ }^{4}$ The iEGG and Animal Biotechnology Center, National Chung Hsing University, Taichung 402, Taiwan.

Received: 10 September 2020 Accepted: 10 March 2021

Published online: 22 March 2021

\section{References}

1. Hernandez-Cabanyero C, Sanjuan E, Fouz B, Pajuelo D, Vallejos-Vidal E, Reyes-Lopez FE, Amaro C. The effect of the environmental temperature on the adaptation to host in the zoonotic pathogen Vibrio vulnificus. Front Microbiol. 2020:11:489. https://doi.org/10.3389/fmicb.2020.00489.

2. Hernandez-Cabanyero C, Lee CT, Tolosa-Enguis V, Sanjuan E, Pajuelo D, Reyes-Lopez F, Tort L, Amaro C. Adaptation to host in Vibrio vulnificus, a zoonotic pathogen that causes septicemia in fish and humans. Environ Microbiol. 2019:21 (8):3118-39. https://doi.org/10.1111/1462-2920.14714.

3. Lo WS, Chen $\mathrm{H}$, Chen CY, Kuo CH. Complete Genome Sequence of Vibrio vulnificus 93U204, a Bacterium Isolated from Diseased Tilapia in Taiwan. Genome Announc. 2014;2(5):e01005-14.

4. Mahmud ZH, Wright AC, Mandal SC, Dai J, Jones MK, Hasan M, Rashid MH, Islam MS, Johnson JA, Gulig PA, Morris JG Jr, Ali A. Genetic characterization of Vibrio vulnificus strains from tilapia aquaculture in Bangladesh. Appl Environ Microbiol. 2010;76(14):4890-5. https://doi.org/1 0.1128/AEM.00636-10.

5. Cheng CL, Huang SJ, Wu CL, Gong HY, Ken CF, Hu SY, Wu JL. Transgenic expression of omega-3 PUFA synthesis genes improves zebrafish survival during Vibrio vulnificus infection. J Biomed Sci. 2015:22(1):103. https://doi. org/10.1186/s12929-015-0208-1. 
6. White PJ, Mitchell PL, Schwab M, Trottier J, Kang JX, Barbier O, Marette A. Transgenic omega-3 PUFA enrichment alters morphology and gene expression profile in adipose tissue of obese mice: potential role for protectins. Metabolism. 2015;64(6):666-76. https://doi.org/10.1016/..meta bol.2015.01.017.

7. McLennan PL, Abeywardena MY, Charnock JS. A comparison of the longterm effects of n-3 and n-6 polyunsaturated fatty acid dietary supplements and the action of indomethacin upon the mechanical performance and susceptibility of the rat heart to dysrhythmia. Prostaglandins Leukot Med. 1987;27(2-3):183-95. https://doi.org/10.1016/0262-1746(87)90070-9.

8. Hardardottir I, Kinsella JE. Increasing the dietary (n-3) to (n-6) polyunsaturated fatty acid ratio increases tumor necrosis factor production by murine resident peritoneal macrophages without an effect on elicited peritoneal macrophages. J Nutr. 1992;122(10):1942-51. https://doi.org/10.1 093/jn/122.10.1942.

9. Apour CS, Bell SJ, Forse RA. Immunologic effects of national cholesterol education panel step-2 diets with and without fish-derived N-3 fatty acid enrichment. JPEN J Parenter Enteral Nutr. 1994;18(4):381-3. https://doi.org/1 $0.1177 / 014860719401800423$

10. Meydani SN. Effect of (n-3) polyunsaturated fatty acids on cytokine production and their biologic function. Nutrition. 1996;12(1 Suppl):S8-14.

11. Whelan J, Gowdy KM, Shaikh SR. N-3 polyunsaturated fatty acids modulate B cell activity in pre-clinical models: implications for the immune response to infections. Eur J Pharmacol. 2016;785:10-7. https://doi.org/10.1016/j.ejphar.2015.03.100.

12. Calder PC. N-3 polyunsaturated fatty acids and immune cell function. Adv Enzym Regul. 1997;37:197-237. https://doi.org/10.1016/S0065-2571(96)00004-0.

13. Nayak S, Al Ashhab A, Zilberg D, Khozin-Goldberg I. Dietary Supplementation with Omega-6 LC-PUFA-Rich Microalgae Regulates Mucosal Immune Response and Promotes Microbial Diversity in the Zebrafish Gut. Biology (Basel). 2020;9(6):119. https://doi.org/10.3390/ biology 9060119 .

14. Wang YD, Peng KC, Wu JL, Chen JY. Transgenic expression of salmon delta5 and delta- 6 desaturase in zebrafish muscle inhibits the growth of Vibrio alginolyticus and affects fish immunomodulatory activity. Fish Shellfish Immunol. 2014;39(2):223-30. https://doi.org/10.1016/j.fsi.2014.04.021.

15. Chiang KY, Lin WC, Tsai TY, Lin CW, Huang SJ, Huang CY, Wu SH, Ken CF, Gong HY, Chen JY, Wu JL. Dual expression of transgenic delta-5 and delta-6 desaturase in tilapia alters gut microbiota and enhances resistance to Vibrio vulnificus infection. PLoS One. 2020;15(7):e0236601. https://doi.org/10.1371/ journal.pone.0236601.

16. Heimes A, Brodhagen J, Weikard R, Seyfert HM, Becker D, Meyerholz MM, Petzl W, Zerbe H, Hoedemaker M, Rohmeier L, Schuberth HJ, Schmicke M, Engelmann S, Kühn C. Hepatic Transcriptome analysis identifies divergent pathogen-specific targeting-strategies to modulate the innate immune system in response to Intramammary infection. Front Immunol. 2020;11:715. https://doi.org/10.3389/fimmu.2020.00715.

17. Salger SA, Reza J, Deck CA, Wahab MA, Baltzegar DA, Murr AT, Borski RJ. Enhanced biodiversity of gut flora and feed efficiency in pond cultured tilapia under reduced frequency feeding strategies. Plos One. 2020;15(7): e0236100. https://doi.org/10.1371/journal.pone.0236100.

18. Yanez JM, Joshi R, Yoshida GM. Genomics to accelerate genetic improvement in tilapia. Anim Genet. 2020;51(5):658-74. https://doi.org/10.1111/age.12989.

19. Cadiz MI, Lopez ME, Diaz-Dominguez D, Caceres G, Yoshida GM, GomezUchida D, Yanez JM. Whole genome re-sequencing reveals recent signatures of selection in three strains of farmed Nile tilapia (Oreochromis niloticus). Sci Rep. 2020;10(1):11514. https://doi.org/10.1038/s41598-020-68064-5.

20. $W u$ SH, Lin HJ, Lin WF, Wu JL, Gong HY. A potent tilapia secreted granulin peptide enhances the survival of transgenic zebrafish infected by Vibrio vulnificus via modulation of innate immunity. Fish Shellfish Immunol. 2018; 75:74-90. https://doi.org/10.1016/j.fsi.2018.01.044.

21. Vinh DC, Mubareka S, Fatoye B, Plourde P, Orr P. Vibrio vulnificus septicemia after handling Tilapia species fish: a Canadian case report and review. Can J Infect Dis Med Microbiol. 2006;17(2):129-32. https://doi.org/10.1155/2006/164681.

22. Horseman MA, Surani S. A comprehensive review of Vibrio vulnificus: an important cause of severe sepsis and skin and soft-tissue infection. Int J Infect Dis. 2011;15(3):e157-66. https://doi.org/10.1016/i.jijd.2010.11.003.

23. Kim BS, Gavin HE, Satchell KJF. Variable Virulence of Biotype 3 Vibrio vulnificus due to MARTX Toxin Effector Domain Composition. mSphere. 2017;2(4):e00272-17. https://doi.org/10.1128/mSphereDirect.00272-17.

24. Wu Y, Xu J, Han X, Qiao G, Yang K, Wen Z, Wen X. Comparative Transcriptome Analysis Combining SMRT- and Illumina-Based RNA-Seq Identifies Potential
Candidate Genes Involved in Betalain Biosynthesis in Pitaya Fruit. Int J Mol Sci. 2020;21(9):3288. https://doi.org/10.3390/ijms21093288.

25. Rhoads A, Au KF. PacBio sequencing and its applications. Genomics Proteomics Bioinformatics. 2015;13(5):278-89. https://doi.org/10.1016/.gpb.2015.08.002.

26. Lindner MS, Strauch B, Schulze JM, Tausch SH, Dabrowski PW, Nitsche A, Renard BY. HiLive: real-time mapping of illumina reads while sequencing. Bioinformatics. 2017;33(6):917-319. https:/doi.org/10.1093/bioinformatics/btw659.

27. The Gene Ontology C. Expansion of the Gene Ontology knowledgebase and resources. Nucleic Acids Res. 2017;45(D1):D331-8.

28. O'Halloran DM. fastQ_brew: module for analysis, preprocessing, and reformatting of FASTQ sequence data. BMC Res Notes. 2017:10(1):275.

29. Lin WC, Chang HY, Chen JY. Electrotransfer of the tilapia piscidin 3 and tilapia piscidin 4 genes into skeletal muscle enhances the antibacterial and immunomodulatory functions of Oreochromis niloticus. Fish Shellfish Immunol. 2016;50:200-9. https://doi.org/10.1016/j.fsi.2016.01.034.

30. Pan CY, Tsai TY, Su BC, Hui CF, Chen JY. Study of the Antimicrobial Activity of Tilapia Piscidin 3 (TP3) and TP4 and Their Effects on Immune Functions in Hybrid Tilapia (Oreochromis spp.). Plos One. 2017;12(1):e0169678.

31. Wu SH, Chou HY, Liu PC, Wu JL, Gong HY. Granulin peptide GRN-41 of Mozambique tilapia is a novel antimicrobial peptide against Vibrio species. Biochem Biophys Res Commun. 2019;515(4):706-11. https://doi.org/10.1016/j.bbrc.2019.06.022.

32. Ting $\mathrm{CH}$, Chen YC, Chen JY. Nile tilapia fry fed on antimicrobial peptide Epinecidin-1-expressing Artemia cyst exhibit enhanced immunity against acute bacterial infection. Fish Shellfish Immunol. 2018;81:37-48. https://doi. org/10.1016/j.ffi.2018.07.008.

33. Moravec AR, Siv AW, Hobby CR, Lindsay EN, Norbash LV, Shults DJ, Symes SJK, Giles DK. Exogenous Polyunsaturated Fatty Acids Impact Membrane Remodeling and Affect Virulence Phenotypes among Pathogenic Vibrio Species. Appl Environ Microbiol. 2017;83(22):e01415-17. https://doi.org/1 0.1128/AEM.01415-17.

34. Dhindsa S, Ghanim H, Batra M, Kuhadiya ND, Abuaysheh S, Green K, Makdissi A, Chaudhuri A, Dandona P. Effect of testosterone on hepcidin, ferroportin, ferritin and iron binding capacity in patients with hypogonadotropic hypogonadism and type 2 diabetes. Clin Endocrinol. 2016;85(5):772-80. https://doi.org/10.1111/cen.13130.

35. Altamura S, Kessler R, Grone HJ, Gretz N, Hentze MW, Galy B, Muckenthaler MU. Resistance of ferroportin to hepcidin binding causes exocrine pancreatic failure and fatal iron overload. Cell Metab. 2014;20(2):359-67. https://doi.org/10.1016/j.cmet.2014.07.007.

36. Nemeth E, Tuttle MS, Powelson J, Vaughn MB, Donovan A, Ward DM, Ganz T, Kaplan J. Hepcidin regulates cellular iron efflux by binding to ferroportin and inducing its internalization. Science. 2004;306(5704):2090-3. https://doi. org/10.1126/science.1104742.

37. Hsieh JC, Pan CY, Chen JY. Tilapia hepcidin (TH)2-3 as a transgene in transgenic fish enhances resistance to Vibrio vulnificus infection and causes variations in immune-related genes after infection by different bacterial species. Fish Shellfish Immunol. 2010;29(3):430-9. https://doi.org/10.1016/j.fsi.2010.05.001.

38. Hannan JP, Laskowski J, Thurman JM, Hageman GS, Holers VM. Mapping the complement factor H-related protein 1 (CFHR1):C3b/C3d interactions. Plos One. 2016;11(11):e0166200. https://doi.org/10.1371/journal.pone.0166200.

39. Abarrategui-Garrido C, Martinez-Barricarte R, Lopez-Trascasa M, de Cordoba SR, Sanchez-Corral P. Characterization of complement factor H-related (CFHR) proteins in plasma reveals novel genetic variations of CFHR1 associated with atypical hemolytic uremic syndrome. Blood. 2009;114(19): 4261-71. https://doi.org/10.1182/blood-2009-05-223834.

40. Sode J, Bank S, Vogel U, Andersen PS, Sorensen SB, Bojesen AB, Andersen MR, Brandslund I, Dessau RB, Hoffmann HJ, et al. Genetically determined high activities of the TNF-alpha, IL23/IL17, and NFkB pathways were associated with increased risk of ankylosing spondylitis. BMC Med Genet. 2018;19(1):165. https://doi.org/10.1186/s12881-018-0680-z.

41. Thurman CE, Rasmussen S, Prestia KA. Effect of 3 euthanasia methods on serum yield and serum cortisol concentration in Zebrafish (Danio rerio). J Am Assoc Lab Anim Sci. 2019;58(6):823-8. https://doi.org/10.30802/AALASJAALAS-18-000144.

42. Rao $X$, Huang $X$, Zhou $Z$, Lin $X$. An improvement of the $2 \wedge(-$ delta delta $C T)$ method for quantitative real-time polymerase chain reaction data analysis. Biostat Bioinforma Biomath. 2013;3(3):71-85.

\section{Publisher's Note}

Springer Nature remains neutral with regard to jurisdictional claims in published maps and institutional affiliations. 